\author{
Costantino-Matteo Fabris \\ (ricercatore di Diritto canonico nell'Università degli Studi Roma Tre, \\ Dipartimento di Giurisprudenza)
}

\title{
La remissione delle censure canoniche. Sviluppo storico e normativa vigente
}

\begin{abstract}
SOMMARIO: 1. Introduzione - 2. L'assoluzione dalle censure nella disciplina precodiciale - 3. (segue) nei trattati canonistici precedenti la codificazione del 1917 - 4. La competenza a concedere l'assoluzione dalle censure nella disciplina precodiciale - 5. La remissione delle censure nel Codex iuris canonici del 1917 - 6. La revisione del Codex del 1917 in tema di remissione delle censure - 7. L'istituto della remissione delle censure nel Codex iuris canonici del 1983 - 8. (segue) la remissione delle censure in foro interno - 9. Le modifiche introdotte dalla costituzione Pascite gregem Dei - 10. Brevi considerazioni conclusive.
\end{abstract}

\section{1 - Introduzione}

La remissione rientra tra le modalità di cessazione della pena canonica assieme alla esecuzione della pena, alla prescrizione (della azione criminale, can. 1362, o della esecuzione della pena, can. 1363), alla abrogazione della legge (can. 1313, § 2) e, ovviamente, alla morte del reo. L'applicazione della pena rimane, in ambito canonico, la soluzione finale ed estrema di un più ampio e complesso percorso di emenda del reo che dovrà intercorrere tra il compimento del fatto delittuoso e l'avvio della procedura penale di tipo amministrativo o giudiziario (cfr. can. 1341). La vigente codificazione canonica prevede dei meccanismi automatici di cessazione della pena per tutte le fattispecie su indicate, a eccezione della remissione che dipende da un atto della competente autorità: assoluzione o dispensa ${ }^{1}$. Dal momento che tutte le pene canoniche, a eccezione di

\footnotetext{
* Contributo sottoposto a valutazione.
}

1 Sulla cessazione delle pene nella vigente normativa si vedano: A. BORRAS, Les sanctions dans l'Église. Commentaire des Canon 1311-1399, Éditions Tardy, Paris 1990, pp. 124-147; V. DE PAOLIS, La cessazione delle pene (cann. 1354-1363), in Il diritto nel mistero della Chiesa, a cura di GRUPPO ITALIANO DOCENTI DI DIRITTO CANONICO, PONTIFICIA UNIVERSiTÀ LATERANENSE, Roma, 1992, 2a ed., vol. III, pp. 496-504; A. CALABRESE, Diritto penale canonico, LEV, Città del Vaticano, 1996, pp. 228-257; D. CITO, La remissione 
quelle espiatorie inflitte per un tempo determinato, sono comminate sempre a tempo indeterminato e sino alla loro remissione, emerge l'importanza dell'istituto oggetto del presente studio quale strumento per la cessazione della pena che non conosce di fatto un corrispettivo negli ordinamenti giuridici secolari ${ }^{2}$.

della pena canonica, in Ius Ecclesiae, 9 (1997), pp. 117-142; V. DE PAOLIS, D. CITO, Le sanzioni nella Chiesa. Commento al Codice di Diritto Canonico Libro VI, Urbaniana University Press, Città del Vaticano, 2000, pp. 257-273; A. BORRAS, Titulus VI De poenarum cessatione. Introducción, in Comentario Exegético al Código de derecho canónico, a cura di Á. MARZOA, J. MiRAS, R. RODRÍGUEZ-OCAÑA, EUNSA, Pamplona, 2002, 3a ed., vol. IV/1, pp. 430-432; D. CITO, voce Absolución de censuras, in Diccionario General de Derecho Canónico [=DGDC], a cura di J. OtADUY, A. VIANA, J. SEDANO, T. Reuters Aranzadi, Cizur Menor, 2012, vol. I, pp. 87-89; A. BORRAS, voce Cesación de la pena, in DGDC, cit., vol. II, pp. 6769; A. BORRAS, voce Remisión de la pena, in DGDC, cit., vol. VI, pp. 897-901; B.F. PIGHIN, Diritto penale canonico, Marcianum Press, Venezia, 2014, 2a ed., pp. 275-294.

2 Nel diritto penale italiano le cause di estinzione del reato e della pena accomunano una serie di istituti tra loro eterogenei. Estinguono il reato: la morte del reo prima della condanna, l'amnistia anteriore alla condanna, la remissione della querela, la prescrizione, l'oblazione, la sospensione condizionale della pena e il perdono giudiziale per i minori. Estinguono la pena: la morte del reo successiva alla condanna, l'amnistia successiva alla condanna, l'indulto, la grazia, la prescrizione della pena, la non menzione della condanna, la liberazione condizionale, la riabilitazione (cfr. G. BETTIOL, Diritto penale. Parte generale, CEDAM, Padova, 1973, 8a ed., pp. 760-792). La distinzione si fonda sulla incidenza che la estinzione ha rispettivamente sulla punibilità astratta o sulla punibilità concreta. La prima sussiste nel momento in cui il reato è completo in tutti i suoi elementi, mentre la seconda si ha solamente nel momento in cui vi sia stata una sentenza di condanna che abbia inflitto una pena (cfr. A. CADOPPI, P. VENEZIANI, Manuale di diritto penale. Parte generale e Parte speciale, CEDAM, Padova, 2007, 3a ed., pp. 507-508). Sulla base di tale distinzione dottrinale nel diritto canonico la remissione della censura sarà sempre causa di estinzione tanto della pena che del reato dal momento che essa avrà incidenza sia sulla punibilità astratta che sulla punibilità concreta. Infatti, anche nel caso di remissione di pena latae sententiae non ancora dichiarata, essendo il reo assoggettato alla pena (in via definitiva) per avere commesso un delitto perfetto in tutti i suoi elementi tipici, il provvedimento di remissione inciderà sia sulla punibilità astratta che su quella concreta. Si dovrà tuttavia tener presente che in realtà il reato, una volta commesso, non potrà estinguersi dal momento che "Ciò che è accaduto non può considerarsi come non accaduto. [...] Nessuna causa di estinzione sarà tale da toglierlo [il reato] dal novero dei fatti accaduti. Si dice allora che si "estingue il reato", vale a dire la qualificazione giuridica del fatto, la sua valutazione negativa compiuta dal legislatore: il fatto viene radiato dal campo del giuridicamente rilevante per essere relegato al mondo della natura» nonostante ciò il reato, ancorché estinto, continuerà a produrre effetti giuridici (incidendo, ad esempio, sulla recidiva o sulla abitualità) ecco che "è doveroso riconoscere che l'estinzione non opera sul reato in toto considerato, ma su di un suo momento sostanziale": G. BETTIOL, Diritto penale. Parte generale, cit., p. 757. 
Il presente contributo intende ripercorrere le tappe salienti relative alla nascita e allo sviluppo dell'istituto, con particolare riferimento alle principali fonti dottrinali dell'età moderna, per poi analizzare la sua sistemazione nel codice del 1917 [=CIC17] e le modifiche apportate dal codice del 1983 e dalla costituzione apostolica Pascite gregem Dei del 23 maggio 2021. Come si avrà modo di vedere, l'istituto dell'assoluzione dalle censure viene disciplinato nel $\mathrm{CIC} 17$ recependo le modalità assolutorie fissate dalla dottrina canonistica soprattutto a partire dal secolo XVI, sia con riferimento alle modalità di concessione della remissione, sia con riguardo alla competenza a concedere tale assoluzione. La nuova codificazione, pur semplificando notevolmente la materia, si pone comunque in linea di continuità con la tradizione, lasciando aperte alcune problematiche relative all'assoluzione dalle censure in foro interno, che da sempre hanno costituito una delle questioni più dibattute dalla dottrina in materia ${ }^{3}$. Le modifiche introdotte da papa Francesco non hanno alterato l'impianto di fondo dell'istituto introducendo però delle specificazioni atte a rendere maggiormente chiaro il dettato normativo con un occhio di riguardo per la riparazione del danno.

\section{2 - L'assoluzione dalle censure nella disciplina pre-codiciale}

Prima della promulgazione del Codex iuris canonici la disciplina relativa all'assoluzione dalle censure appariva particolarmente articolata e si fondava su di una serie assai eterogenea di norme, come emerge anche dalla lettura delle fonti utilizzate per la redazione dei relativi canoni del CIC17 ${ }^{4}$. Nel diritto canonico che si fondava sulle decretali mancava, infatti,

${ }^{3}$ Cfr. R. MAZZOLA, La pena latae sententiae nel diritto canonico. Profili comparati di teoria generale, CEDAM, Padova, 2002, pp. 303-335.

4 Si veda: Codex Iuris Canonici Pii X Pontifici Maximi iussu digestus Benedicti Papae XV auctoritate promulgatus. Praefatione, fontium annotatione et indice analytico-alphabetico ab. Em.mo Petro Card. Gasparri auctus, Typis Polyglottis Vaticanis, Romae, 1917, in particolare pp. 605-606, 610-612, 622-623. Per una più generale ricostruzione della evoluzione del diritto penale canonico, con indicazione delle fonti principali, si vedano: P. HINSCHIUS, System des Katholischen Kirchenrechts, mit besonderer Rücksicht auf Deutschland, I. von Guttentag, Berlin, 1888, vol. IV, pp. 691-864; P. HINSCHIUS, System des Katholischen Kirchenrechts, cit., Berlin, 1895, vol. V; P. HINSCHIUS, System des Katholischen Kirchenrechts, cit., Berlin, 1897, vol. VI; L. KAHN, Étude sur le délit et la peine en droit canon, A. Crépin-Leblond, Nancy, 1898; J.J. CHRIST, Dispensation From Vindicative Penalties. An Historical Conspectus and Commentary, The Catholic University of America Press, Washington DC, 1943, pp. 14-51; F.X. WERNZ, P. VIDAL, I us canonicum, apud aedes 
una trattazione sistematica delle tematiche penalistiche di natura generale, dal momento che le disposizioni de criminibus et poenis si concentravano sull'esame dei singoli delitti5; tale impostazione era acuita anche dalla decisa prevalenza, quanto meno a partire dalla metà del secolo XVI, dei manuali di Institutiones morales le quali, a loro volta, avevano dato luogo, per l'applicazione pratica, al genere letterario dei "casi di morale" (specialmente in ambito teologico-morale) ${ }^{6}$, per cui l'attenzione di canonisti e teologi si concentrava sulle fattispecie criminose/peccaminose e sui metodi per porvi rimedio. Da tale forma mentis derivava un sostanziale disinteresse per i temi di carattere generale, quale è quello della assoluzione in sé considerata ${ }^{7}$.

Una prima trattazione dell'istituto della assoluzione dalla censura e dei passaggi necessari per essere riammessi alla piena comunione con la Chiesa è contenuta nel libro V delle decretali di Gregorio IX sotto il titolo De sententia excommunicationis ove viene presentata un'ampia casistica di reati e delle relative riserve in tema di assoluzione ${ }^{8}$. Sino alle soglie dell'età moderna, la natura della pena è vista quasi esclusivamente in un'ottica vendicativa, motivo per cui il tema dell'assoluzione rimaneva in subordine rispetto al tema, decisamente preminente, della tipologia di pena stabilita per ogni singolo delitto in vista del recesso del reo dalla

Universitatis Gregorianae, Romae, 1951, 2a ed., pp. 3-13; G. MICHIELS, De delictis et poenis. Commentarius Libri V Codicis Juris Canonici, Desclée, Parisiis-Tornaci-Romae-Neo Eboraci, 1961, vol. I, pp. 30-40; L. GEROSA, La scomunica è una pena? Saggio per una fondazione teologica del diritto penale canonico, Éditions Universitaires, Fribourg Suisse, 1984, pp. 403-428.

5 Cfr. D. SCHIAPPOLI, Diritto penale canonico, in Enciclopedia del diritto penale italiano. Raccolta di monografie, a cura di E. PESSINA, Società Editrice Libraria, Milano, 1905, vol. I, pp. 613-614 e pp. 647-650; G. MICHIELS, De delictis et poenis, cit., vol. I, pp. 37-39.

${ }^{6}$ La letteratura in materia è sterminata. Si rimanda, da ultimo, all'opera monumentale di M. VIDAL, Historia de la teología moral. V. De Trento al Vaticano II. 1. Crisis de la razón y rigorismo moral en el Barroco (s. XVII), Editorial El Perpetuo Socorro, Madrid s.d.e., in particolare pp. 43-254, e M. VIDAL, Historia de la teología moral. V. De Trento al Vaticano II. 2. El siglo de la Ilustración y la moral católica (s. XVIII), Editorial El Perpetuo Socorro, Madrid s.d.e., in particolare pp. 775-1030.

7 Cfr. sul punto A. VITALE, voce Delitti. e) Diritto canonico, in Enciclopedia del diritto, Giuffrè, Milano, 1964, vol. XII, pp. 29-38, sul punto p. 31.

8 X 5.39 De sententia excommunicationis, in E. FRIEDBERG, Corpus iuris canonici. Editio Lipsiensis secunda. Pars secunda, Akademische Druk-U. Verlagsanstalt, Graz, 1959, coll. 889 ss. Sulla scomunica medievale si veda, almeno, E. VODOLA, Excommunication in the Middle Age, University of California Press, Berkeley-Los Angeles-London, 1986. 
contumacia9 ${ }^{9}$ Data poi la commistione esistente tra la sfera giuridica e quella morale, spesso l'assoluzione veniva concessa nell'ambito del foro penitenziale specie per gli aspetti riguardanti le materie spirituali ${ }^{10}$. Tale dualismo caratteristico del diritto canonico medievale e comportante una sostanziale commistione tra fori, penitenziale e giudiziario, si protrarrà sino alle soglie della contemporaneità con conseguente sviluppo della teologia morale quale scienza concorrente con quella giuridica.

Una specifica trattazione relativa all'assoluzione dalle censure è rinvenibile nell'opera di Francisco Suárez, il quale dedica al tema un'intera disputatio del suo De censuris (1 $1^{\text {a }}$ ed. 1603) ${ }^{11}$. Per comprendere appieno il significato e l'importanza che il teologo-giurista spagnolo attribuisce all'istituto dell'assoluzione si deve tener presente che, a differenza di quanto sostenuto da una parte della canonistica precedente, per Suárez la censura ha finalità medicinali oltre che vendicative ${ }^{12}$. In questo senso il

9 Il concetto di contumacia in diritto canonico fa riferimento all'atteggiamento del delinquente il quale, nonostante legalmente ammonito dalla competente autorità, persiste nel delitto o, pur desistendo da esso, rifiuta di pentirsi, di risarcire il danno o di riparare lo scandalo. Nel caso di censure latae sententiae, si ha contumacia del delinquente per il fatto stesso di avere commesso un delitto perfetto in tutti i suoi elementi; in questo secondo caso la contumacia cesserà quando il delinquente, oltre ad avere interrotto la condotta criminosa, abbia anche dato prova di effettivo pentimento e abbia almeno seriamente promesso adeguata riparazione del danno o dello scandalo. In entrambi i casi la contumacia ha origine nel disprezzo (contemptus) del delinquente nei confronti della sanzione o dell'autorità che l'ha comminata e anche dell'eventuale ammonizione (monitio) che precede un provvedimento di natura sanzionatoria. Cfr. F. DELLA ROCCA, La contumacia nel diritto canonico. Appunti di esegesi critica sulle fonti, Edizioni Universitarie, Roma, 1943; P. TORQUEBIAU, voce Contumacia, Contumax, in Dictionnaire de droit canonique, a cura di R. NAZ, Librairie Letouzey et Ané, Paris, 1949, t. IV, coll. 506-541, in particolare coll. 540-541; T.P. CUNNINGHAM, The contumacy required to incur censures, in The Irish Theological Quarterly, 21 (1954), pp. 332-356; G. MICHIELS, De delictis et poenis. Commentarius Libri V Codicis Juris Canonici, Desclée, Parisiis-Tornaci-Romae-Neo Eboraci, 1961, vol. III, pp. 18-35.

10 Tra gli innumerevoli studi relativi allo sviluppo della distinzione tra delitto e peccato si vedano: P. PRODI, Una storia della giustizia. Dal pluralismo dei fori al moderno dualismo tra coscienza e diritto, il Mulino, Bologna, 2000, in particolare pp. 211-218; pp. 265268 e pp. 448-454; P. ERDÖ, Il peccato e il delitto: la relazione tra due concetti fondamentali alla luce del diritto canonico, Giuffrè, Milano, 2014.

11 Si tratta della disputatio VII, De absolutionis et modis quibus censura tolli potest, in F. SUÁREZ, De censuris, disp. VII, in Opera omnia, tomus XX, apud S. Coleti, Venetiis, 1749, pp. 105-137.

12 "Censura est poena spiritualis et medicinalis, privans usu aliquorum spirituali bonorum, per Ecclesiasticam potestatem ita imposita, ut per eandem ordinarie absolvi possit", F. SUÁREZ, De censuris, disp. I, sect. I, cit., p. 7. In realtà Suárez non fu il primo 
doctor eximius ha cura di precisare che, sebbene il fine principale della censura sia quello di privare di un bene spirituale per un determinato periodo colui che si rende colpevole di determinati reati ${ }^{13}$, tale pena comprende nondimeno due fini ulteriori: un fine temporale, che consiste nel patimento di un danno commisurato al danno causato, in un'ottica strettamente retribuzionistica; e un fine spirituale, che consiste, secondo quanto indicato da S. Paolo, nel consentire al reo, mediante l'espiazione in questo mondo delle proprie colpe, di "essere salvato nel giorno del Signore" $(1 \text { Cor } 5,5)^{14}$.

Proprio per fare risaltare le finalità medicinali della censura, fra i tre modi attraverso cui questa può venir meno, particolare rilevanza assume l'assoluzione $^{15}$. Sebbene l'assoluzione dalla censura possa essere concessa anche in foro interno, essa costituisce comunque un atto proprio della potestas iurisdictionis. Per ottenere l'assoluzione è necessario che il reo non solo abbia fatto emenda recedendo dalla contumacia ma anche che si sia pentito del reato commesso ${ }^{16}$. Condizioni ribadite da Suárez, anche laddove affronta il tema delle solennità da osservare per ottenere l'assoluzione dalla censura, con la precisazione che per ottenere l'assoluzione in foro esterno il delinquente, oltre a dovere manifestare "sufficiens signum oboediendi" 17 avrebbe dovuto anche recedere dalla

autore ad attribuire un fine medicinale alla censura, essendo tale finalità in parte già presente nel diritto delle decretali cfr.: L. KAHN, Étude sur le délit et la peine en droit canon, cit., pp. 60-81; J.L. SANTOS DIEZ, Fin medicinal de la censura hasta Suárez, in Revista Española de Derecho Canónico, 6 (1951), pp. 571-650, in particolare pp. 619-628.

${ }^{13}$ F. SUÁREZ, De censuris, disp. VI, sect. I, cit., p. 98, il teologo-giurista granadino non concepisce la possibilità che la censura rimanga comminata a tempo indefinito senza che vi sia possibilità di emenda per il reo: tale circostanza renderebbe infatti del tutto vana la finalità medicinale della pena.

14 "Hujusmodi autem in primis est observantia obedientiae Ecclesiasticae: deinde reparatio spiritualis lapsus commissi ab eo, qui censura ligatur, ut nunc a peccato resurgat, et tandem spiritus ejus salvus fiat in die Domini, ut dicitur I. ad Corinth. 5", F. SUÁREZ, De censuris, disp. VI, sect. I, cit., p. 98. Sulla evoluzione storica del diritto penale canonico, con particolare riferimento alle fonti relative al primo millennio tra $i$ molti studi si vedano: D. SCHIAPPOLI, Diritto penale canonico, cit., pp. 625-642; G. MICHIELS, De delictis et poenis, cit., vol. I, pp. 30-36; T. JOUBERT, La responsabilité canonique de l'Église en matière pénale. Regard historique, des temps apostoliques jusqu'à la chute de Rome, in L'Année Canonique, 51 (2009), pp. 237-252.

${ }^{15}$ Le altre due modalità indicate sono la morte del reo e il decorso dei termini nella censura imposta per un tempo determinato.

${ }^{16}$ F. SUÁREZ, De censuris, disp. VI, sect. I, cit., pp. 106-107.

${ }^{17}$ F. SUÁREZ, De censuris, disp. VII, sect. X, cit., p. 136. 
contumacia, mentre in caso di richiesta di assoluzione in foro interno era solamente richiesta la manifestazione di obbedienza nei confronti dell'autorità ecclesiastica ${ }^{18}$. Sebbene in passato all'assoluzione dalla censura fossero legate numerose solennità di natura giuridica sia concomitanti alla concessione dell'assoluzione sia a essa successive, Suárez ricorda come le prime fossero all'epoca cadute in desuetudine, mentre le seconde si rendevano necessarie o meno a seconda del tipo di censura e della necessità di dare adeguata pubblicità dell'avvenuta assoluzione. Il doctor eximius sottolinea che tali solennità, specialmente quelle susseguenti alla concessione dell'assoluzione, erano sconsigliate laddove l'assoluzione fosse avvenuta in forum conscientiae, dal momento che le assoluzioni occulte dovevano rimanere tali, essendo sufficiente la testimonianza del confessore ${ }^{19}$.

Sebbene nell'opera di Suárez siano già contenuti tutti gli elementi fondamentali che caratterizzeranno l'istituto dell'assoluzione dalla censura, tuttavia la prima trattazione sistematica del diritto penale canonico può essere considerata, anche per il tema attinente al presente studio, quella del gesuita Carlo Antonio Tesauro ${ }^{20}$. Nella prima parte del suo De poenis ecclesiasticis praxis absoluta et universalis (Typis L. Grignani, Romae, 1640, $1^{\text {a }}$ ed.), relativa alla dottrina generale circa le pene ecclesiastiche latae sententiae, Tesauro indica i criteri distintivi tra dispensa e assoluzione ${ }^{21}$. Quanto all'assoluzione, essa: è un atto di giustizia (cfr. can.

18 F. SUÁREZ, De censuris, disp. VII, sect. X, cit., p. 137.

19 Va specificato che per forum conscientiae si intende qui il foro extrasacramentale, per cui il termine confessore è utilizzato per distinguere il giudice ecclesiastico dal semplice sacerdote competente a rimettere la censura. Per meglio comprendere la distinzione operata da Suárez tra il foro interno sacramentale ed extrasacramentale si deve fare riferimento a F. SUÁREZ, De legibus ac Deo legislatore, 1. 8, c. 6, nn. 13-15, in Opera omnia, tomus V, apud S. Coleti, Venetiis, 1740, pp. 465-466. Per un'analisi del pensiero del teologo-giurista granadino sul punto, si veda: C.-M. FABRIS, Foro interno. Genesi ed evoluzione dell'istituto canonistico, Mucchi, Modena, 2020, pp. 87-88.

${ }^{20}$ Cfr. D. SCHIAPPOLI, Diritto penale canonico, cit., p. 651. L'opera di Tesauro, che ebbe diverse edizioni con l'aggiornamento di U. Giraldi, è citata da Wernz, nel suo Ius decretalium, come testo di riferimento nella sezione De poenis ecclesiasticis in genere. Carlo Antonio Tesauro (Torino, 1587- Roma, 1655), gesuita, insegna diritto civile e canonico a Pisa e Torino e poi teologia morale; ricoprì per vent'anni la carica di penitenziere minore della BasilicaVaticana. Cfr. C. SOMMERVOGEL, voce Thesaurus, Tesauro, Charles Antoine, in Bibliothèque de la Compagnie de Jésus, O. Schepens-A. Picard, Bruxelles-Paris, 1896, t. VII, col. 1968.

$21 \mathrm{Si}$ è scelto di riportare tra parentesi i canoni del CIC17 che in qualche modo discendono dalla trattazione di Tesauro, per fare meglio comprendere l'influenza che essa ebbe sulla codificazione canonica e pure i nomi di alcuni autori cui Tesauro si rifà 
2248, § 2); ha ad oggetto solamente le pene medicinali (cfr. can. 2236, § 1) e comunque viene concessa per le pene che non siano perpetue; per essere concessa necessita che sia cessata la contumacia (cfr. can. 2248, § 2) previo venir meno della ratio scandali; non si può negare l'assoluzione al reo pentito, essendo sufficiente che questi dia prova di avere riparato il danno causato; contro la mancata assoluzione si può ricorrere al superiore per denegata giustizia; l'assoluzione può essere concessa dal vescovo (cfr. cann. 2236-2237); per concedere l'assoluzione è sufficiente godere della potestas iurisdictionis; una volta concessa non può essere revocata; se viene meno la causa per la quale l'assoluzione fu concessa non viene meno il provvedimento di assoluzione; l'assoluzione può essere concessa solamente ai propri sudditi ratione domicilii (Suárez, Barbosa, Sanchez) e non anche in ragione del quasi domicilio o della provenienza. In virtù delle concessioni fatte dal Concilio tridentino l'assoluzione in foro interno poteva essere concessa anche in ragione del quasi domicilio e ai girovaghi22; le disposizioni riguardanti l'assoluzione vanno interpretate nel senso più favorevole al reo (cfr. can. 2219).

Per quanto invece concerne la dispensa essa, sempre secondo Tesauro, è un atto di grazia; ha ad oggetto le pene perpetue; richiede una causa giusta e razionale per essere concessa; essendo la dispensa un atto di grazia può essere negata al reo per motivi gravi (grave scandalo, grave necessità pubblica, motivi di giustizia o carità) e va concessa con sobrietà; contro il provvedimento che nega la dispensa non si può proporre ricorso; la dispensa deve essere concessa da chi ha adottato il provvedimento punitivo; per concedere una dispensa è necessario essere stati a ciò espressamente delegati; una volta concessa può essere sempre revocata da colui che l'ha concessa in virtù di un atto di potestà ordinaria; secondo parte degli autori (ad esempio T. Sánchez) se viene meno la causa per la quale fu concessa la dispensa, questa si dovrà revocare (contra, ad esempio, F. Suárez); una volta ottenuta la dispensa questa non avrà più valore se il soggetto reitererà il delitto per il quale l'aveva ottenuta (cfr. can. 2288 CIC17); le disposizioni riguardanti la dispensa sono soggette a interpretazione stretta ${ }^{23}$.

rispetto a specifiche questioni.

${ }^{22}$ L'assoluzione dalle censure aventi a oggetto l'amministrazione del sacramento della penitenza e dell'eucaristia e la loro ricezione (come, ad esempio, la scomunica o l'interdetto personale) poteva essere concessa anche in sede di pellegrinaggio, a patto che questo non avvenisse fraudolentemente al solo scopo di ottenete l'assoluzione dalla censura.

${ }^{23}$ C.A. TESAURO, De poenis ecclesiasticis praxis absoluta et universalis, Typis Seminarii, 
Come si vede l'esposizione di Tesauro fu sostanzialmente ripresa nelle disposizioni del CIC17 relative alla remissione ${ }^{24}$. Gli autori successivi presero infatti spunto, oltre che dal diritto penale romano, dalla trattazione sistematica del gesuita piemontese anche se spesso il tema della remissione occupò piuttosto i trattati di teologia morale. A titolo di esempio ci si può riferire all'opera di Reiffenstuel il quale, proprio in apertura della parte del suo trattato relativo a de poenitentiis et remissionibus, ha cura di specificare che: "Verum quia haec materia penitus Theologica est, et de hac Theologi ex professo tractant, nil de ea hic agentes lectorem ad Theologos, et nostram Theologiam Moralem remittimus ..." 25 . In effetti, se si passa alla lettura della Theologia moralis dello stesso autore si trova menzione, dopo la definizione delle censure e delle modalità di inflizione delle stesse, della disciplina prevista per l'assoluzione dalle medesime. Non erano previste specifiche modalità per la assoluzione ("Nulla determinata forma verborum requiritur ad tollendas Censuras, sed sufficit, quibuscumque verbis voluntas absolventis sufficienter significetur"26) essendo eventualmente utile fare cenno, nel pronunciare la formula assolutoria, ai motivi che avevano portato alla censura e a quelli che ne giustificavano la remissione ${ }^{27}$. Nel

Patavii, 1761, caput XX, pp. 15-16. Alle soglie della prima codificazione canonica Wernz riprende quasi alla lettera la trattazione di Tesauro laddove indica le modalità di estinzione delle censure, cfr. F.X. WERNZ, Ius decretalium, t. VI, Ius poenale Ecclesiae catholicae, Officina Libraria Giachetti, Prati, 1913, p. 177, nota 144. Wernz inoltre, più correttamente di quanto non faccia il Codex, il quale si riferisce alla sola remissione della pena (can. 2236), parla di estinzione dei delitti e delle pene, in ciò abbracciando le teoriche fatte proprie sul punto dalla scienza giuridica secolare, cfr. F.X. WERNZ, Ius decretalium, t. VI, Ius poenale Ecclesiae catholicae, cit., p. 90.

${ }^{24} \mathrm{Si}$ veda sul punto: ANONIMO, Il nuovo Codice di Diritto canonico (Libro V). Principii del diritto penale, in La Civiltà Cattolica, 69-3 (1918), pp. 490-501, in particolare pp. 493-495.

${ }^{25}$ A titolo di esempio ci si può riferire a: A. REIFFENSTUEL, Jus canonicum universum, apud D. Ercole, Romae, 1834, t. V, n. 1, p. 383.

26 A. REIFFENSTUEL, Theologia moralis, J.B. Albritii, Hieronymi filii, veneti typographi, Mutinae, 1758, t. II, dist. I, quaestio III, nn. 41-43, p. 5, in particolare n. 41. Circostanza ribadita anche da Ferraris: "Ita etiam Communis Doctorum. Forma absolutionis a censuris non habet praefixa verba, sed potest dari verbis exprimentibus absolutionem talis specialis Censurae, a qua fit absolutio", L. FERRARIS, voce Censura, in Prompta bibliotheca, canonica, juridica, moralis, theologica, apud J.-P. Migne Editorem, Parisiis, 1861, t. II, n. 42, col. 478.

27 Vi erano al contempo numerose modalità di assoluzione che non potevano essere utilizzate a pena di illiceità o di invalidità, cfr. L. FERRARIS, voce Absolutio, in Prompta bibliotheca, canonica, juridica, moralis, theologica, apud J.-P. Migne Editorem, Parisiis, 1863, t. I, coll. 158-160. 
caso di sospensione inflitta per un tempo determinato o, ancora, sottoposta a determinate condizioni, essa sarebbe cessata con il semplice decorso del tempo o a seguito del compimento della condizione imposta e ciò valeva anche per i casi di interdetto ${ }^{28}$. Nei casi ordinari di censura era invece necessario ottenere l'assoluzione, vuoi in foro esterno vuoi in foro interno, da parte di un soggetto avente la facoltà di concedere l'assoluzione $^{29}$ e previa cessazione della contumacia da parte del reo $^{30}$. Per ciò che riguardava più specificatamente la scomunica si richiedeva poi apposita sentenza di assoluzione anche nel caso di recessione dalla contumacia e soddisfazione ${ }^{31}$.

\section{3 - (segue) nei trattati canonistici precedenti la codificazione del 1917}

I trattati di diritto canonico precedenti alla codificazione già indicavano, tra le modalità di cancellazione delle censure, le seguenti: "Delentur autem censurae septem modis, abrogatione, revocatione, cassatione, morte ferentis, morte notati, lapsu temporis et absolutione" 32 , modalità che erano

28 "Si Suspensio fuit lata ad determinatum dumtaxat tempus v.g. ad unum, aut duos annos, vel usque ad positionem certae conditionis v.g. dicendo, Sis suspensus, donec satisfeceris: tunc elapso illo tempore, adimpleta conditione, utputa praestita satisfactione per se ipsam cessat Suspensio, neque opus est alia formula absolutionis, sive sententia restitutionis", A. REIFFENSTUEL, Theologia moralis, cit., t. II, n. 42, p. 5.

29 "Caeterum extra hosce casus necesse est, adhiberi veram absolutionem a Censuris, ab habente legitimam facultatem absolvendi datam", A. REIFFENSTUEL, Theologia moralis, cit., t. II, n. 42 , p. 5 .

$30 \mathrm{Si}$ fa riferimento alla necessità del recesso dalla contumacia al fine dell'ottenimento della assoluzione già in un testo di Innocenzo IV riportato nel Liber Sextus: "Secus autem, si se contumacem confiteatur, vel alias de contumacia sua constet, quia forte praedixerat in iudicio, quod minime compareret. Tunc enim, quum manifesta sit contumacia, et manifesta reputetur offensa, non est ei, nisi primo expensarum satisfactione ac de stando iudicio cautione praestita, absolutio impendenda", VI 5.11.7, in E. FRIEDBERG, Corpus iuris canonici. Editio Lipsiensis secunda. Pars secunda, Akademische Druk-U. Verlagsanstalt, Graz, 1959, col. 1100.

31 "Excommunicatio major semel contracta, etiam cessante contumacia excommunicati, et praestita satisfactione, non tollitur sine absolutione", A. REIFFENSTUEL, Theologia moralis, cit., t. II, dist. II, quaestio IV, appendix ad. I, p. 14. Affermazione sostanzialmente identica si rinviene in P. LAYMANN, Theologia moralis in $V$ Libros distributa, pars I, Typographia Remondiniana, Venetiis, 1760 (1625, 1ª ed.), tract. V, cap. VII, p. 41.

32 C.S. BERARDI, Commentaria in jus ecclesiasticum universum, Sumptibus L. Rossi, Mediolani, 1847, vol. II, pars. II, diss. III, cap. XI, pp. 350-351. Ojetti parla di cessazione 
da ritenersi concorrenti tra loro, a seconda dei casi. Per cancellare le censure comminate ferendae sententiae si poteva procedere mediante abrogazione, revocazione o cassazione del provvedimento contenente la censura $^{33}$, mentre l'assoluzione in senso stretto era la modalità di cancellazione prevista per rimettere le censure latae sententiae ${ }^{34}$. Per concedere l'assoluzione si richiedeva che il reo, dopo avere abbandonato la contumacia, avesse mostrato un vero pentimento e, per quanto concerneva lo scandalo o il danno causato, avesse almeno dimostrato il serio proposito di riparare a essi risarcendo, per quanto possibile, le eventuali parti lese ${ }^{35}$. Le modalità di concessione dell'assoluzione variavano dunque a seconda che la pena fosse comminata latae sententiae o ferendae sententiae. Nel primo caso il delinquente, una volta avuta contezza di essere incorso in una pena latae sententiae, aveva l'onere di ricorrere alla competente autorità per ottenere l'assoluzione. Nel caso invece di pena inflitta a seguito di procedura giudiziaria si aprivano, come visto, diverse

della censura piuttosto che di cancellazione, cfr. B. OJETTI, Synopsis rerum moralium et iuris pontificii, ex Officina Polygraphica Editrice, Romae, 1909, 3a ed., vol. I, coll. 695-706.

33 Per tali modalità si vedano le voci di E. MAGNIN, voce Abrogation de la loi, in Dictionnaire de droit canonique, a cura di R. NAZ, Librairie Letouzey et Ané, Paris, 1935, t. I, coll. 115-120, e di A. VILLIEN, voce Absolution, in Dictionnaire de droit canonique, cit., t. I, coll. 120-123, in particolare col. 122 nonché di A. BRIDE, voce Censures (peines), in Dictionnaire de droit canonique, Librairie Letouzey et Ané, Paris, 1942, t. III, coll. 169-233, in particolare coll. 214-230.

${ }^{34}$ Come segnala ad esempio M. LEGA, Praelectiones in textum iuris canonici de iudiciis ecclesiasticis, De iudiciis criminalibus in genere et in specie. De delictis et poenis praemisso tractatu, Typis Vaticanis, Romae, 1899, libro II, vol. III, pp. 176-177.

${ }^{35}$ Sui requisiti necessari per ottenere l'assoluzione si veda: F. SCHMALZGRUEBER, Jus ecclesiasticum universum, ex Typographia Rev. Camera Apostolicae, Romae, 1845, t. V, pars II, pars IV, tit. XXXIX, nn. 100-111, pp. 420-424, il quale sottolinea in particolare la necessità del risarcimento della parte lesa al fine dell'ottenimento dell'assoluzione. D'Annibale spiega che: "absolutio ei solum danda est qui eam poscit humiliter; et dummodo recesserit a contumacia; scandalum, propter quod censurae subjacebat, reparaverit; et, si quem laeserat, satisfecerit parti laesae; vel, si praesenti satisfacere nequit, promiserit, cum primum poterit satisfacturum. Ad haec, si crimen sit de atrocioribus, jurare debet (nisi forte sit impubes), se numquam in illud relapsurum", G. D'ANNIBALE, Summula theologiae moralis ad usum seminarii reatini, Typis S. Trinchi, Reate, 1874, pars I, liber III, tract. VI, n. 310, p. 192. Il dettato del can. 2242, § 3, del CIC17 era meno rigido in quanto stabiliva che, per dare prova di recesso dalla contumacia, fosse sufficiente fornire "congruam satisfactionem pro damnis et scandalo... aut saltem serio promiserit"; norma ripresa quasi letteralmente dal can. 1347, § 2, CIC83 e oggi resa più restrittiva alla luce delle modifiche introdotte dal nuovo can. $1361, \S 4$, di cui si dirà più oltre. 
vie per ottenere l'assoluzione ${ }^{36}$. Questa poteva essere concessa dal giudice di secondo grado nel caso in cui si fosse accertato che la sentenza era stata chiaramente ingiusta. Nel caso in cui la sentenza di primo grado fosse invece ritenuta fondata, l'appellante era invitato a chiedere l'assoluzione al giudice di prime cure, dando prova di serio pentimento e di volere riparare il danno. Infine, in caso di dubbio sulla validità della pena comminata in primo grado, il giudice di appello poteva scegliere se concedere egli stesso l'assoluzione o rinviare l'appellante avanti il giudice di primo grado affinché quest'ultimo potesse disporre l'assoluzione decorso un determinato periodo di tempo ${ }^{37}$. L'appello, nel caso delle censure, non aveva normalmente effetto sospensivo rispetto al provvedimento punitivo inflitto dal giudice di primo grado, essendo comunque facoltà dell'appellante richiedere il beneficio della absolutio ad cautelam $^{38}$. In questo caso il giudice d'appello aveva la facoltà di sospendere temporaneamente la sanzione comminata in primo grado qualora avesse preliminarmente valutato una possibile nullità della pena per dubium facti o dubium iuris ${ }^{39}$.

36 Una descrizione sintetica di tali procedure è contenuta in: G. PÉRIES, La procédure canonique moderne dans les causes disciplinaires et criminelles. Notions pratiques sur le tribunaux ecclesiastiques et le fonctionnement des officialités, A. Roger et F. Chernoviz, Paris, 1898, pp. 267-269.

37 Tale procedura era stata determinata da Benedetto XIII, Additiones ad XII et XIII Decretum Clementis VIII: "Quoad absolutionem ultimatam a censuris servetur dispositio supradicti decreti 12. Clementis VIII, juxta etiam canonicam sanctionem, quod in casu constet de justitia censurarum, debeat remitti absolutio danda ad judicem a quo; si vero constet clare de injustitia, judex ad quem absolutionem impendat. Si vero adsit dubietas, an fuerit justa, vel injusta, honestius esset, ut ad excommunicatorem infra aliquem competentem terminum praefigendum absolvendus remittatur, licet etiam judex ad quem hoc casu idipsum per se praestare jure possit", in Concilium romanum in Sacrosancta Basilica Lateranensi celebratum Anno Universalis Jubilaei MDCCXXV, Typographia Rocchi Bernabò, Romae, 1725, p. 211.

$38 \mathrm{Su}$ particolari forme di absolutio si veda: M. SCHMOECKEL, L'absolutio ab instantia. Son développement en Europe et ses implications constitutionnelles, in Revue d'Histoire des Facultés de Droit et de la Science Juridique, 19 (1998), pp. 173-187. Sulle fonti dell'istituto canonistico si veda: R. HELMHOLZ, The Spirit of classical Canon Law, University of Georgia Press, Athens (Georgia)-London, 1996, p. 376, il riferimento è, in primis, a Innocenzo III in X 2.20.38.

39 SACRA CONGREGAZIONE DEI VESCOVI E DEI REGOLARI, Decretum De appellationibus, 16 ottobre 1600, § 13, in Collectanea in usum Secretariae Sacrae Congregationis Episcoporum, et regularium, a cura di A. BIZZARRI, ex Tipographia Rev. Camerae Apostolicae, Romae, 1863, pp. 20-22: "Absolutio ad cautelam nonnisi parte citata, et visis actis, cum dubitatur de nullitate excommunicationis ab homine prolatae, vel a jure si occurrat dubium facti vel probabile dubium juris concedenda erit", in particolare p. 22. 
Tra le opere che costituiscono degli immediati antecedenti delle disposizioni poi trasfuse nella futura codificazione canonica rientrano certamente i lavori di Hollweck e Pillet. Hollweck - in linea con quanto già fatto da Tesauro il quale aveva esposto la materia in ordine alfabetico - dà conto del diritto penale vigente secondo una trattazione di tipo sistematico organizzata sul modello dei moderni codici penali ${ }^{40}$. Pillet, poi, dedica un certo numero di articoli proprio alla assoluzione dalla censura, ricordando che: "Licet censurae sint poenae medicinales, tamen statim non cessant ex correctione rei, sed necessario dari debet absolutio: haec correctio est tantum motivum legitimum ad dandam absolutionem" 41 . Pillet specifica che tale assoluzione potrà essere concessa tanto in foro interno, nei casi di peccato o censura occulta, sia in foro esterno, nei casi di censura notoria e pubblica, descrivendo poi in pochi e sintetici articoli la competenza e le modalità richieste per concedere tale assoluzione, riprendendo sul tema le trattazioni di diritto canonico precedentit ${ }^{42}$.

\section{4 - La competenza a concedere l'assoluzione dalle censure nella disciplina pre-codiciale}

Il tema dell'assoluzione dalle censure era strettamente collegato a quello della competenza a concedere tale assoluzione ${ }^{43}$. Il Concilio di Trento aveva stabilito la riserva in capo al pontefice, per la Chiesa universale, e ai vescovi, ciascuno nella propria diocesi, relativamente alle "causas aliquas criminum graviores [...] praesertim quoad illa, quibus excommunicationis censura adnexa est" ${ }^{\prime \prime}$; riserva valida non solamente in foro esterno ma

40 J. HOLLWECK, Die kirchliche Strafgesetze. Zusammengestellt und kommentiert, F. Kirchheim, Mainz, 1899.

41 A. PILLET, Jus canonicum generale distributum in articulos, P. Lethielleux, Parisiis, 1900, 2a ed., pp. 386-387.

42 Si confrontino a titolo d'esempio: F. SCHMALZGRUEBER, Jus ecclesiasticum universum, cit., t. V, pars IV, tit. XXXIX, § 1 de censuris in genere, nn. 1-101, pp. 384-421; G. D'ANNIBALE, Summula theologiae moralis ad usum seminarii reatini, cit., pars I, nn. 287289 e nn. 305-315, pp. 176-178 e pp. 188-194.

43 Una sintesi storica sulla competenza circa la remissione delle censure è contenuta in: R. ASPE, El "caso urgente" para la remisión de las censuras en la normativa canónica actual. Estudio histórico-canónico y aplicación práctica del can. 1357 del Código de Derecho Canónico, in Periodica, 101 (2012), pp. 159-198, in particolare pp. 162-179.

44 Concilio di Trento, Sessione XIV, 25 novembre 1551, cap. VII, testo rinvenibile in $\mathbf{H}$. DENZINGER, A. SCHÖNMETZER, Enchiridion symbolorum definitionum et declarationum de rebus fidei et morum, Herder, Barcinonae-Friburgi Brisgoviae-Romae, 1976, 36ª ed., p. 
anche in foro interno. L'assoluzione da qualsiasi peccato o censura poteva essere concessa, sempre in base alle previsioni tridentine, da qualsiasi sacerdote allorquando il richiedente si fosse trovato in articulo mortis ${ }^{45}$. Tale potestà era implicitamente riconosciuta anche ai prelati "habentes jurisdictionem quasi Episcopalem" così come ammesso a più riprese dalla Sacra Congregazione del Concilio ${ }^{46}$, potestà che nel tempo venne estesa anche ai superiori degli ordini regolari ${ }^{47}$.

Affinché si potesse parlare di crimen gravius erano necessarie almeno tre caratteristiche: essere internamente o esternamente grave; essere completo e consumato nella sua specie; essere certamente riservato, in quanto la materia, essendo odiosa, era soggetta a interpretazione stretta (disposizione poi confluita nel can. 19 del CIC17).

L'assoluzione dalle censure poteva essere di due specie: privata, quando si dava nel foro interno, solenne, quando si concedeva nel foro esterno. La facoltà di concedere l'assoluzione era di competenza di colui che aveva dichiarato con sentenza la censura (o del suo successore) o di un suo delegato. In caso di censura prevista da una legge (ad esempio, censura latae sententiae) spettava al vescovo o al confessore la facoltà di concedere l'assoluzione ${ }^{48}$. Non tutti i sacerdoti o confessori possedevano la facoltà necessaria per concedere l'assoluzione, essendo previsto, come detto, un sistema di riserve sia in capo al Pontefice che ai vescovi.

I casi riservati si potevano suddividere in diverse categorie: ex parte reservantis, in papali, episcopali e regolari a seconda della competenza a concedere l'assoluzione; ex parte modi, ratione gravitatis (o directa) o ratione censurae (o indirecta) in base alla gravità del delitto o alla tipologia di pena prevista.

397, n. 1687. Il Concilio tridentino aveva previsto una serie di altre riserve per specifici reati le quali sono tutte riportate all'interno della costituzione Apostolicae Sedis del 12 ottobre 1869, in Acta Sanctae Sedis, 5 (1869-70), pp. 320-324 e pp. 327-329.

${ }^{45}$ Concilio di Trento, Sessione XIV, 25 novembre 1551, cap. VII, in H. DENZINGER, A. SCHÖNMETZER, Enchiridion symbolorum, cit., p. 397, n. 1688.

${ }^{46} \mathrm{Si}$ veda sul punto quanto riportato da BENEDETTO XIV, De synodo dioecesana, t. I, Kirchheim-Schott et Thielmann, Moguntiae, 1842 (dalla editio secunda Mechliniensis), libro V, cap. V, n. II, pp. 331-332.

${ }^{47}$ Una sintesi delle competenze ad assolvere in singoli e specifici casi e dei relativi divieti, nonché delle modalità di assoluzione è contenuta nelle voci Absolvere e Censura in L. FERRARIS, Prompta bibliotheca, canonica, juridica, moralis, theologica, cit., t. I, coll. 131166 e t. II, coll. 467-484.

${ }^{48}$ Cfr. G. MORONI, voce Assoluzione dalle censure, in Dizionario di erudizione storicoecclesiastica, Tipografia Emiliana, Venezia, 1840, vol. III, pp. 78-80, in particolare p. 78. 
L'enumerazione dei singoli delitti riservati era contenuta in una serie di documenti pontifici. Quanto ai reati riservati al pontefice ratione censurae, questi erano individuati fino al 1770 nelle bolle In Coena Domini ${ }^{49}$ e, successivamente, nelle costituzioni di Pio IX Apostolicae Sedis (12 ottobre $1869)^{50}$ e Romanus Pontifex (28 agosto 1873) ${ }^{51}$ e di Leone XIII Officiorum ac munerum (25 gennaio 1897)52, nonché nella Istruzione della S. Congregazione dei Vescovi e Regolari dell'11 giugno 188053. Tali documenti, che rimasero in vigore sino alla promulgazione del $\mathrm{CIC} 17^{54}$,

${ }^{49}$ I cui contenuti vennero definitivamente fissati nella costituzione Pastoralis di Urbano VIII del 1627. Sulla storia e sui contenuti della bolla In Coena Domini si veda: F. CLAEYS BOUAERT, voce Bulle In Coena Domini, in Dictionnaire de Droit Canonique, a cura di R. NAZ, Librairie Letouzey et Ané, Paris, 1937, t. II, coll. 1132-1136.

50 Il testo originale è rinvenibile in Acta Sanctae Sedis, 5 (1869-70), pp. 305-331. Numerosi gli studi a commento della costituzione, tra i più significativi si vedano: G. D'ANNIBALE, In constitutionem Apostolicae Sedis qua censurae latae sententiae limitantur. Commentarii, Pacelli Tomassini, Terni, 1873 (ripubblicato in più edizioni sino al 1909); G. BUCCERONI, Commentarius de constitutione Apostolicae Sedis secundum omnes decisiones sacrarum rom. Congregationum, Ex Typographia Polyglotta, Romae, 1886; A. AMANIEU, voce Apostolicae Sedis, in Dictionnaire de Droit Canonique, cit., t. I, coll. 698-724. Il testo della costituzione, con le integrazioni operate dalla Romanus Pontifex del 1873 è rinvenibile in appendice al volume di F.X. WERNZ, Ius decretalium, t. VI, Ius poenale Ecclesiae catholicae, cit., pp. 457-470. Riporta un elenco dei delitti riservati al pontefice e ai vescovi in periodo pre-codiciale: J.L. SÁNCHEZ-GIRÓN RENEDO, Evolución de la disciplina canónica en materia de absolución y remisión de pecados y censuras reservadas durante los últimos cien años, in Ciencia Tomista, 137 (2010), pp. 139-171, in particolare pp. 148-150 e p. 157. Una comparazione tra le norme contenute nella Apostolicae Sedis e nel CIC17 è rinvenibile in: G.L. LEECH, A comparative study of the Constitution "Apostolicae Sedis" and the "Codex Juris Canonici", Catholic University of America, Washington DC, 1922.

${ }^{51}$ Testo in Pii IX Pontificis Maximi acta. Pars prima, ex Typographia Vaticana, Romae, s.d.e., vol. 6, pp. 239-246. Testo rinvenibile anche in appendice al volume di P. GASPARRI, Codicis iuris canonici fontes, Typis Polyglottis Vaticanis, 1933, vol. III, n. 565, pp. 74-77.

52 Testo in Acta Sanctae Sedis, 29 (1896-97), pp. 388-400.

53 Testo in Acta Sanctae Sedis, 13 (1880), pp. 324-336. Tale Istruzione, indirizzata ai vescovi dei territori ricompresi nel neonato Regno d'Italia, era volta a stabilire norme comuni per lo svolgimento dei procedimenti disciplinari e criminali secondo la forma economica (modum procedendi oeconomice) nei confronti di chierici; si tratta di una primigenia forma di procedura di tipo amministrativo penale. Cfr. L. MUSSELLI, Il contributo di Joseph Hollweck alla codificazione del diritto penale canonico, in M. VISMARA MISSIROLI, L. MUSSELLI, Il processo di codificazione del diritto penale canonico, CEDAM, Padova, 1983, p. 122.

54 Una serie di altri decreti, emanati in anni successivi, stabilivano particolari disposizioni in riferimento a casi specifici di impossibilità a recarsi presso l'autorità 
stabilivano da un lato quali fossero le censure riservate al pontefice 'in via ordinaria', speciali modo e specialissimo modo, ma anche quali fossero le scomuniche riservate agli ordinari nonché quelle non riservate ${ }^{55}$.

Le censure si suddividevano in latae a iure e latae ab homine ${ }^{56}$. La competenza ad assolvere dalle censure a iure era determinata, in base alla ratio censurae, secondo una serie di criteri stabiliti nei summenzionati documenti ${ }^{57}$. Per le censure $a b$ homine la competenza era riservata a colui che l'aveva inflitta, o al suo successore o superiore nei casi particolari, mentre poteva essere rimessa da qualsiasi confessore nei casi generali se non fossero stabilite particolari riserve in base al tipo di censura ${ }^{58}$.

Nei casi di pericolo di morte imminente la riserva veniva meno, almeno in via temporanea, come su accennato; tale disposizione, ribadita autorevolmente dal Concilio tridentino, aveva in realtà origine nel Liber Sextus di Bonifacio VIII, con la specificazione che, in caso di cessato

competente a concedere l'assoluzione. Ad esempio: decreti del S. Uffizio 23 giugno 1886, in Acta Sanctae Sedis, 19 (1886), pp. 46-47; 9 novembre 1898 in Analecta Ecclesiastica, 7 (1899), p. 6; 7 giugno 1899 in Analecta Ecclesiastica, 7 (1899), p. 339; 5 settembre 1900, in Analecta Ecclesiastica, 8 (1900), p. 489; 19 ottobre 1900 in Analecta Ecclesiastica, 9 (1901), p. 54 .

55 Il codice del 1917 disciplinò nuovamente il sistema delle riserve stabilendo il numero delle censure riservate all'ordinario e a soggetti a lui equiparati (sei); il numero delle censure riservate simplici modo alla Sede Apostolica (tredici), quelle a questa riservate speciali modo (dodici) e specialissimo modo (quattro). L'elenco completo con la relativa spiegazione delle singole fattispecie è rinvenibile, oltre che nei vari manuali redatti dai commentatori del CIC17, anche in P. CERATO, Censurae vigentes ipso facto a Codice Iuris Canonici excerptae, Typis Seminarii, Patavii, 1921, 2a ed., pp. 89-192.

56 Per pene a iure si intendevano quelle determinate dalla legge o da un precetto penale, mentre per pene $a b$ homine si intendevano quelle inflitte mediante sentenza o mediante provvedimento amministrativo, cfr. P. CIPROTTI, Pene. VI. Diritto canonico, in Enciclopedia del diritto, vol. XXXII, Giuffrè, Milano, 1982, pp. 856-864, in particolare p. 858.

57 Una sintesi di tali criteri è contenuta in: T. ORTOLAN, Censures ecclésiastiques, in Dictionnaire de théologie catholique, Letouzey et Ané, Paris, 1905, t. II, coll. 2113-2136, in particolare coll. 2132-2133.

58 Una sintesi di tali disposizioni è contenuta in T. LARUMBE Y LANDER, Disciplina vigente sobre absolucion de censuras y pecados reservados, in La Cientia Tomista, 1-3 (1910), pp. 406-416, in particolare p. 408; 1-4 (1910), pp. 55-6. Elenchi di reati canonici con l'indicazione della riserva di cui erano oggetto sono contenuti in numerosi trattati; a titolo di esempio si segnalano: C.A. TESAURO, De poenis ecclesiasticis praxis absoluta et universalis, cit., pp. 46 ss.; C. RONCAGLIA, Universa moralis theologia ad usum confessarium, ex Officina Josephi Justi, Lucae, 1833, tomo II, pp. 24 ss.; J. HOLLWECK, Die kirchliche Strafgesetze zusammengestellt und kommentiert, cit., §§ 95-301. 
pericolo, si rendeva necessario ottenere l'assoluzione da parte della competente autorità ${ }^{5}$.

\section{5 - La remissione delle censure nel Codex iuris canonici del 1917}

Nel codice del 1917 l'istituto della remissione era disciplinato in via generale nei cann. 2236-2240. I canoni relativi alla remissione traevano sostanzialmente spunto, come visto, non solo dalle norme contenute nel Corpus iuris canonici e dalle disposizioni successive dei pontefici, ma anche dalla sistemazione teorica di Suárez e dalla manualistica dell'Ottocento. È lo stesso Pillet, nel suo commento pubblicato all'indomani dell'entrata in vigore del CIC17, a sottolineare che i canoni relativi alla remissione "ne font que reproduire les prescriptions du droit ancien, sans changement appréciable"60.

Il can. 2236, in particolare, distingueva tra remissione di censura e remissione di pena vendicativa, da attuarsi la prima mediante assoluzione e la seconda mediante concessione di dispensa ${ }^{61}$. La differente disciplina relativa alla remissione era quindi demandata da un lato ai cann. 22482254 per l'assoluzione dalle censure e ai cann. 2289-2290 per la estinzione

59 "Eos, qui a sententia canonis vel hominis, quum ad illum, a quo alias de iure fuerant absolvendi, nequeunt propter imminentis mortis articulum aut aliud impedimentum legitimum pro absolutionis beneficio habere recursum, ab alio absolvuntur, si, cessante postea periculo vel impedimento huiusmodi, se illi, a quo his cessantibus absolvi debebant, quam cito commode poterunt, contempserint praesentare, mandatum ipsius super illis, pro quibus excommunicati fuerant ...", VI 5.11.22, in E. FRIEDBERG, Corpus iuris canonici. Editio Lipsiensis secunda. Pars secunda, cit., col. 1105. La rigidità della riserva era mitigata anche in caso di impossibilità, per il reo pentito, di recarsi personalmente dinanzi all'autorità competente a rimettere la censura.

60 A. PILLET, Introduction à l'étude du Code canonique. Des Changements apportés à la Législation antécédente, Librairie Catholique Emmanuel Vitte, Lyon-Paris, 1919, 2a ed., p. 101.

${ }^{61}$ Il CIC83 ha sostituito alle pene vendicative quelle espiatorie (can. 1312, § 2); si tratta comunque di sanzioni che hanno quale obiettivo quello della riparazione del delitto, laddove le pene medicinali hanno per scopo l'emenda del reo. Sulla modifica in questione si veda in particolare: P. CIPROTTI, Elementi di novità nel diritto penale canonico vigente, in Monitor Ecclesiasticus, 114 (1989), pp. 17-28, in particolare p. 21. Sulla distinzione tra pene vendicative e censure nel CIC17, ma con osservazioni valide anche per l'attuale codificazione si veda: P. CIPROTTI, voce Censura. b) Censure ecclesiastiche, in Enciclopedia del diritto, Giuffrè, Milano, 1960, vol. VI, pp. 734-738, in particolare p. 734; P. CIPROTTI, voce Pene. VI. Diritto canonico, cit., pp. 856-864. 
delle pene vendicative. Come segnalato ${ }^{62}$ tale scelta poggiava sulla diversa natura dell'atto di remissione: nel caso delle censure esso rappresentava un atto di giustizia (absolutio) il cui diniego era eventualmente ricorribile; per ciò che concerneva le pene vendicative, invece, la remissione era un atto di grazia (dispensatio) ${ }^{63}$.

Tale duplice disciplina aveva differenti risvolti in relazione alla remissione in foro interno. Il can. 2251 prevedeva che l'assoluzione dalle censure riguardasse principalmente il foro esterno, ma era comunque possibile ottenere la remissione dalla censura anche in foro interno (sia sacramentale che extrasacramentale), se ciò fosse stato fatto in vista della salus animae del reo.

La principale problematica riguardava gli effetti della remissione. Se questa veniva concessa in foro esterno, essa produceva i propri effetti in entrambi i fori, sia esterno che interno. Nel caso, invece, di remissione ottenuta nel foro interno, tale atto dava diritto al soggetto assolto di comportarsi come tale, avendo cura di non creare scandalo ${ }^{64}$. Il senso della precisazione appare evidente: non essendovi la possibilità di dare prova certa del provvedimento assolutorio ottenuto in foro interno nel caso in cui il fatto delittuoso fosse stato notorio, ciò avrebbe potuto creare scandalo tra i fedeli.

A tal proposito il can. 2251 specificava che, se non si potesse dare prova in foro esterno della remissione ottenuta o questa non si potesse almeno legittimamente presumere, il superiore competente avrebbe potuto comunque richiedere, al soggetto cui la censura fosse già stata rimessa in foro interno, il rispetto della censura fin quando egli non avesse ottenuto la remissione anche nel foro esterno. La censura, quindi, già rimessa in foro interno, continuava a produrre i suoi effetti nel foro esterno, con evidente conflitto tra i due fori in favore di quest'ultimo.

Pillet non mancò di sottolineare alcune difficoltà conseguenti alla scelta, operata dal legislatore, circa il modo di concedere la remissione delle censure. Se, infatti, era chiaro che la concessione in foro interno

62 Cfr. D. CITO, La remissione della pena canonica, cit., p. 122.

63 Cfr. F.X. WERNZ, P. VIDAL, Ius canonicum, tomus VII, Ius poenale ecclesiasticum, apud Aedes Universitatis Gregorianae, Romae, 1951, 2a ed., p. 233.

64 Come è noto, la ratio scandali costituisce uno dei cardini del diritto penale canonico, tanto che l'antigiuridicità del fatto va valutata avendo presente tale caratteristica (si vedano, ad esempio, gli attuali cann. 1341, 1344, 1347, 1352) ed essa è motivo di operatività del discusso can. 1399 laddove si prevede la punibilità di comportamenti che siano potenzialmente idonei a creare scandalo anche se non immediatamente sanzionabili in base a una apposita previsione normativa. 
dovesse avvenire mediante l'assoluzione sacramentale e, dunque, con la pronuncia delle relative formule, meno chiara era la modalità da utilizzare per le remissioni in foro esterno, dal momento che:

"Dans le second cas, il y a une autre formule, dont certainement beaucoup de prêtres ignorent l'existence, bien qu'elle soit contenue dans le Rituel romain (canon 2250, § 3). Tout cela est bien compliqué et a besoin de quelques explications, surtout pour nous, en France, qui avons laissé tomber en désuétude tout ce qui concerne les jugements et les peines du for externe"65.

Constatazione di una desuetudine che derivava dall'avere demandato gran parte dei giudizi sulle questioni di natura penale all'ambito della teologia morale piuttosto che a quello del diritto.

Il CIC17 si concentrava poi sui soggetti competenti ad assolvere dalle censure (can. 2253), lasciando comunque una certa libertà di assoluzione al confessore in casi urgenti determinati dalla impossibilità di osservare la censura latae sententiae senza pericolo di scandalo grave o di infamia o se durum sit poenitenti in statu gravis peccati permanere per il tempo necessario affinché provveda all'assoluzione il superiore competente (can. 2254). In caso di assoluzione in periculo mortis concessa da un sacerdote privo delle speciali facoltà necessarie per tale scopo $^{66}$, si richiedeva al soggetto cui la censura fosse stata rimessa di rivolgersi in un momento successivo alla competente autorità per ottenere la remissione dalla censura $\mathrm{o}$, comunque, affinché venisse stabilita l'entità della pena nel caso di censura a iure67.

La disciplina della remissione delle pene vendicative in foro interno era invece stabilita dal can. 2290. Il canone sanciva la possibilità, per qualsiasi confessore, di sospendere, in foro interno sacramentale, l'obbligazione di osservare la pena vendicativa latae sententiae nel caso in

65 A. PILLET, Introduction à l'étude du Code canonique, cit., pp. 107-108. Ma si veda anche F. CAPPELLO, Tractatus Canonico-Moralis de censuris, Marietti, Taurini-Romae, 1950, 4a ed., pp. 87-88 ove vengono riportate le relative formule assolutorie.

66 Si trattava, in sostanza, di tutti i soggetti indicati nel can. 882, ovvero di tutti coloro che avessero ottenuto l'ordine sacro ancorché privi delle facoltà per confessare. Si veda sul punto: F. CAPPELLO, Tractatus Canonico-Moralis de censuris, cit., pp. 101-105. Più in generale sui casi straordinari di assoluzione dalle censure si veda F.E. MORIARTY, The extraordinary absolution from censures. An historical synopsis and commentary, The Catholic University of America, Washington DC, 1938.

67 Il can. 2217 del CIC17 ribadiva la distinzione tra pene $a$ iure e pene $a b$ homine. Le prime erano determinate dalla legge stessa, le seconde erano imposte con precetto particolare o con sentenza giudiziale di condanna. 
cui l'osservanza della pena fosse causa di infamia o scandalo per il reo. In questo caso il confessore avrebbe comunque dovuto imporre l'obbligo di ricorrere al vescovo o alla Penitenzieria entro un mese per ottenere la remissione. In casi straordinari poteva essere lo stesso confessore a concedere la dispensa richiesta imponendo la penitenza normalmente prevista per casi analoghi.

Il can. 2232, § 1, CIC17 stabiliva dunque che le pene latae sententiae, sia medicinali che vendicative, obbligavano il delinquente in entrambi i fori (in utroque foro). Come noto alcune pene medicinali comportavano la esclusione dalla comunione ecclesiale, con conseguente impedimento alla ricezione dei sacramenti (come ad esempio la scomunica - can. 2260 - o l'interdetto personale - can. 2275 -); in questo caso il reo non poteva essere assolto dal peccato senza essere stato precedentemente assolto dalla censura, a norma del can. 2250, § 2 .

Come visto il can. 2254, § 1 , prevedeva che, in casi più urgenti, per evitare scandalo o infamia derivanti dalla osservanza esteriore della censura o per il caso in cui fosse duro per il reo rimanere in stato di peccato durante il tempo necessario affinché il superiore competente provvedesse a rimettere la censura, il confessore potesse assolvere dalle censure latae sententiae in foro interno; rimanendo l'obbligo in capo al penitente assolto di rivolgersi entro un mese alla Penitenzieria o al superiore competente per ottenere l'assoluzione. La remissione della censura concessa nel foro interno era quindi pienamente efficace e produttiva di effetti giuridici in questo foro e, sempre in tale foro, veniva eliminato l'obbligo di osservare la pena prevista per il reato commesso ${ }^{68}$.

Ma il can. 2251 conteneva in realtà una riserva: qualora nella comunità si producesse uno scandalo, il reo, già assolto in foro interno, non potendo dare prova dell'assoluzione ottenuta nel foro interno sacramentale, avrebbe comunque dovuto osservare la pena nel foro esterno: ecco che si creava un conflitto tra foro esterno e foro interno che comportava il venir meno del principio della certezza giuridica proprio dei moderni ordinamenti69. L'ormai ex reo, infatti, si sarebbe trovato nella

68 Cfr. sul punto: F.E. MORIARTY, The extraordinary absolution from censures. An historical synopsis and commentary, cit., pp. 142-218.

69 Cfr. sul punto M. ARTECHE, Observaciones sobre lo jurídico y lo moral en el ordenamiento canónico. (A propósito de un libro), in Ius Canonicum, 1 (1961), pp. 473-474; R. CASTILLO LARA, Algunas reflexiones sobre la futura reforma del libro $V$ C.I.C., in Salesianum, 23 (1961), pp. 317-339, in particolare pp. 336-337. Si vedano anche le osservazioni svolte da V. DE PAOLIS, Animadversiones ad "Schema documenti quo disciplina sanctionum seu poenarum in Ecclesia latina denuo ordinatur», in Periodica, 63 (1974), 
condizione di dovere comunque rispettare le limitazioni imposte dalla pena nonostante gli effetti di quest'ultima fossero già stati rimossi dal provvedimento di remissione ottenuto in foro interno.

Il conflitto tra foro esterno e foro interno era determinato dal fatto che quanto avviene in ambito sacramentale, essendo coperto dal segreto confessionale, non consentiva di provare l'ottenimento del provvedimento concesso dall'autorità nel foro interno, con la conseguenza che la potestas iurisdictionis pure legittimamente esercitata nel foro interno e produttiva di veri effetti giuridici, non potendo essere provata, risultava di fatto limitata e dava vita a possibili incertezze. Tanto Cappello che Roberti indicavano la possibilità, per superare tale incertezza di natura probatoria, che il confessore, con il consenso del reo, rilasciasse una dichiarazione scritta della remissione della censura concessa in foro interno, dal momento che una dichiarazione di questo tipo non avrebbe violato il sigillo sacramentale. Infatti, mentre quando il confessore esercita il munus di assolvere dai peccati, egli agisce qua Deus, nell'assolvere dalla censura agisce in qualità di minister Ecclesiae e, dunque, di pubblico ufficiale: tale azione non sarebbe dunque coperta dal sigillo ${ }^{70}$.

Il problema risultava risolto alla radice allorquando i casi accennati venivano riferiti al foro interno extrasacramentale, dove il problema della prova non si poneva e, di conseguenza, era possibile provare l'avvenuta remissione della censura quando ve ne fosse stata necessità.

\section{6 - La revisione del Codex del 1917 in tema di remissione delle censure}

Sebbene il Concilio Vaticano II non abbia direttamente affrontato il tema relativo al diritto penale nella Chiesa, è pur vero che è a partire dalle critiche emerse in quegli anni che si iniziò a porre mano alla revisione del libro V del CIC17. Tra il 1966 e il 1967, terminato il Concilio, la Pontificia Commissione per la revisione del codice, istituita sin dal 196371, elaborò

pp. 489-507; V. DE PAOLIS, «Totum ius poenale ad externum tantum forum limitatum est», in Periodica, 65 (1976), pp. 297-315.

${ }^{70}$ F. ROBERTI, De delictis et poenis, apud custodiam librariam Pontificii Instituti Utriusque Iuris, Romae, 1944, 2a ed., vol. I, pars II, pp. 353-354; F. CAPPELLO, Tractatus Canonico-Moralis de censuris, cit., p. 95.

71 Si vedano i documenti relativi in Communicationes, 1 (1969), pp. 35-38. Una ricostruzione dei lavori preparatori della nuova codificazione canonica è rinvenibile in: $F$. D'OSTILIO, La storia del nuovo codice di diritto canonico. Revisione - Promulgazione Presentazione, LEV, Città del Vaticano, 1983, pp. 23-31; G. FELICIANI, Dal Codice del 1917 
una serie di principi che avrebbero dovuto servire da linee guida per $\mathrm{i}$ lavori di revisione. La Commissione Centrale dei Consultori licenziò all'unanimità il 3-8 aprile 1967 i Principia quae Codicis iuris canonici recognitionem dirigant i quali furono successivamente approvati dal I Sinodo dei Vescovi tenutosi a Roma tra il 30 settembre e il 4 ottobre del 196772. In essi si specificava, tra l'altro, che era necessario coordinare al meglio le interazioni tra i due fori, al fine di evitare possibili conflitti, in particolar modo per ciò che riguarda il diritto sacramentale e quello penale, nonché la necessità di limitare a poche e specifiche fattispecie le pene latae sententiae ${ }^{73}$.

La scelta operata dal legislatore fu quella di mantenere, nel codice del 1983, un apposito libro dedicato al diritto penale superando così sia le obiezioni più radicali che pretendevano addirittura l'abolizione del diritto penale canonico ${ }^{74}$, sia le posizioni di quanti avrebbero voluto limitare il diritto penale al solo foro esterno ${ }^{75}$.

al Codice del 1983, in S. FERRARI (a cura di), Il nuovo Codice di diritto canonico. Aspetti fondamentali della codificazione postconciliare, il Mulino, Bologna, 1983, pp. 23-35; J.L. GUTIÉRREZ, La formazione dei Principi per la riforma del «Codex iuris canonici», in I Principi per la revisione del Codice di diritto canonico. La ricezione giuridica del Concilio Vaticano II, a cura di J. CANOSA, Giuffrè, Milano, 2000, pp. 5-29, in particolare pp. 5-13.

72 Il testo dei Principia è in Communicationes, 1 (1969), pp. 77-85 e in appendice a F. D'OSTILIO, La storia del nuovo codice di diritto canonico, cit., pp. 113-119. Per la prima impostazione dei lavori riguardanti la revisione del diritto penale si vedano: $\mathbf{P}$. CIPROTTI, De iure poenali recognoscendo, in Communicationes, 2 (1970), pp. 99-107, e Brevis conspectus de labore hucusque a Commissione peracto deque peragendo, in Communicationes, 6 (1974), pp. 33-35.

${ }^{73} \mathrm{Su}$ questi specifici temi si vedano: J.M. POMMARÈS, Le deuxième principe pour la réforme du droit canonique du Synode des Évêques de 1967, la coordination des fors dans le droit canonique revisité trente ans après, in I Principi per la revisione del Codice di diritto canonico, cit., pp. 103-126, e V. DE PAOLIS, L'attuazione della riforma del diritto penale canonico, in I Principi per la revisione del Codice di diritto canonico, cit., pp. 669-708. Ricostruiscono l'iter dei lavori della commissione che si occupò della riforma del diritto penale canonico: $\mathbf{P}$. CIPROTTI, Qualche punto caratteristico della riforma del diritto penale canonico, in Studi in memoria di Mario Petroncelli, Jovene, Napoli, 1989, vol. I, pp. 131-145, e M. VENTURA, Pena e penitenza nel diritto canonico postconciliare, ESI, Napoli, 1996, pp. 11-26. Proposte di riforma in parte recepite dal dettato codiciale sono quelle formulate da: A. MOSTAZA RODRIGUEZ, De foro interno iuxta canonistas postridentinos, in Acta Conventus Internationalis canonistarum, Romae, diebus mai 1968 celebrati, a cura di PONTIFICIA COMmissio Codici IURIS CANONICI ReCOGNOSCENDO, Typis Polyglottis Vaticanis, 1970, pp. 269-294.

${ }_{74}$ Per una ricostruzione delle critiche mosse al diritto penale canonico tra gli anni cinquanta e settanta del Novecento si vedano V. DE PAOLIS, De legitimitate et opportunitate poenarum latae sententiae in iure poenali canonico, in Periodica, 62 (1973), pp. 
Nella sottocommissione che si occupò di elaborare gli schemi del nuovo libro VI si discusse circa la necessità di limitare il diritto penale canonico al solo foro esterno, in linea con i Principia poco sopra richiamati. In questo senso si propose di circoscrivere gli effetti di scomunica e interdetto al fine di non inibire la possibilità di ricevere i sacramenti della riconciliazione e dell'unzione degli infermi a quanti vi fossero incorsi ${ }^{76}$. Il

319-373; F.E. ADAMI, Continuità e variazioni di tematiche penalistiche nel nuovo Codex iuris canonici, in Ephemerides Iuris Canonici, 40 (1984), pp. 55-136, in particolare pp. 56-98; M. VENTURA, Pena e penitenza nel diritto canonico postconciliare, cit., pp. 11-64; J. BERNAL, Aspectos del derecho penal canónico. Antes y depués del CIC de 1983, in Ius Canonicum, 49 (2009), pp. 373-412, in particolare pp. 373-385; J.M. CABEZAS CAÑAVATE, Una mirada histórico canónica al devenir del derecho penal canónico desde 1917 hasta nuestros días (I parte), in Ephemerides Iuris Canonici, 56 (2016), pp. 219-244, in particolare pp. 226-238. L'errata impostazione di quanti sostenevano, nel post-concilio, la non necessarietà di un diritto penale per la Chiesa è stata rimarcata da papa Francesco nel riformare il libro VI del CIC83 laddove afferma che: "In passato, ha causato molti danni la mancata percezione dell'intimo rapporto esistente nella Chiesa tra l'esercizio della carità e il ricorso - ove le circostanze e la giustizia lo richiedano - alla disciplina sanzionatoria. Tale modo di pensare - l'esperienza lo insegna - rischia di portare a vivere con comportamenti contrari alla disciplina dei costumi, al cui rimedio non sono sufficienti le sole esortazioni o i suggerimenti. Questa situazione spesso porta con sé il pericolo che con il trascorrere del tempo, siffatti comportamenti si consolidino al punto tale da renderne più difficile la correzione e creando in molti casi scandalo e confusione tra i fedeli. È per questo che l'applicazione delle pene diventa necessaria da parte dei Pastori e dei Superiori. La negligenza di un Pastore nel ricorrere al sistema penale rende manifesto che egli non adempie rettamente e fedelmente la sua funzione [...] Invero la carità richiede che $\mathrm{i}$ Pastori ricorrano al sistema penale tutte le volte che occorra, tenendo presenti i tre fini che lo rendono necessario nella comunità ecclesiale, e cioè il ripristino delle esigenze della giustizia, l'emendamento del reo e la riparazione degli scandali", FRANCESCO, costituzione apostolica Pascite gregem Dei, 23 maggio 2021, in L'Osservatore Romano, 161122 (1 giugno 2021), pp. 2-3, in particolare p. 3.

75 Sul punto, ad esempio, A. SCHEUERMANN, Erwägungen zur kirchlichen Strafrechtsreform, in Archiv für katholisches Kirchenrecht, 131 (1962), pp. 393-415; R.A. STRIGL, Das Funktionsverhaeltnis zwischen kirchlichen Strafgewalt und Oeffentilichkeit, M. Hueber Verlag, München, 1965; F.R. MCMANUS, The internal forum, in Acta Conventus Internationalis canonistarum, cit., pp. 251-261. Anche la così detta canonistica laica italiana tendeva per la maggior parte a escludere che le questioni di foro interno avessero a oggetto la sfera giuridica ma riguardassero piuttosto quella morale, per tutti si veda $\mathbf{S}$. LARICCIA, Giurisdizione ecclesiastica, in Enciclopedia del diritto, Giuffrè, Milano, 1970, vol. XIX, pp. 469-489, in particolare pp. 470-471.

${ }^{76} \mathrm{Si}$ veda in proposito il testo del can. 16, § 1, riportato in Communicationes, 9 (1977), p. 147 secondo il quale: "Excommunicatio vetat: $[\ldots]$ b) sacramenta, exceptis paenitentia et infirmorum unctione, recipere, et sacramenta vel sacramentalia conficere vel administrare". Proposta poi trasfusa nel can. 1331, § 1, n. 2, in seguito alle modifiche introdotte sia dal Coetus studiorum che in sede di Schema codicis recognitum (can. 1282, §1, 
dibattito sul punto fu ampio sia in dottrina che da parte dei soggetti chiamati a esprimere il loro giudizio sul primo schema di revisione del diritto penale ${ }^{77}$.

Nel 1977, specialmente alla luce delle osservazioni giunte dalle conferenze episcopali, si decise di ritornare al divieto, già presente nel CIC17, per i soggetti colpiti da scomunica o interdetto di ricevere tutti i sacramenti, prevedendo però la possibilità di remissione delle censure nel foro interno sacramentale perché

"sarebbe troppo duro per lo scomunicato, soprattutto nel caso di delitti occulti puniti con pena latae sententiae, differire fino al momento dell'ottenimento della remissione della pena in foro esterno la ricezione del sacramento della penitenza e quindi l'acquisizione della grazia"78.

In questo senso si stabilì, come poi sancito dal can. 1357, § 1, la possibilità di rimettere in foro interno sacramentale le sole censure latae sententiae non dichiarate dal momento che, in caso di pena dichiarata, sarebbe stato necessario ottenere la remissione nel foro esterno ${ }^{79}$.

\section{7 - L'istituto della remissione delle censure nel Codex iuris canonici del 1983}

A differenza di quanto avveniva nella precedente codificazione il CIC83 tratta l'istituto della remissione in un unico titolo (il VI del libro sesto) senza distinguere tra la remissione delle pene medicinali e di quelle espiatorie, sebbene da parte della dottrina si sia ritenuto che la nuova

\section{n. 2).}

77 Per la dottrina si deve richiamare in particolare il dibattito tra De Paolis e Coccopalmerio in Periodica, 63 (1974), Periodica, 64 (1975) e Periodica, 65 (1976), mentre per le altre osservazioni si può vedere quanto scritto dal Coetus studiorum de iure poenali nella Brevis relatio de animadversionibus generalibus, in Communicationes, 7 (1975), pp. 94-95.

78 "in schemate praevideri debet norma pro remissione excommunicationis (vel interdicti) in foro interno sacramentali eo quod nimis durum esset excommunicato, praesertim in delictis occultis poena l.s. mulctatis, usque ad obtentam remissionem poenae in foro externo receptionem sacramenti paenitentiae et proinde acquisitionem gratiae differre", in Communicationes, 9 (1977), p. 322.

79 A. BORRAS, Titulus VI De poenarum cessatione. c. 1357 comentario, in Comentario Exegético al Código de derecho canónico, cit., vol. IV/1, pp. 444-445. 
trattazione non abbia in realtà fatto venir meno la distinzione, esistente in precedenza, tra le due tipologie di pene ${ }^{80}$.

Nel caso di una pena medicinale, infatti, il superiore competente rimetterà la pena quando il reo abbia cessato la contumacia ai sensi del can. 1358, mentre nel caso di pena espiatoria il provvedimento del superiore avrà il carattere di grazia, non essendo egli obbligato a rimettere la pena prima del tempo previsto. Nel primo caso, essendo venuta meno la contumacia del reo, la pena ha esaurito il suo scopo e, quindi, l'atto di remissione è un atto di giustizia che si concreta in un obbligo in capo al superiore di concedere il provvedimento di remissione.

I canoni 1354-1361 riguardano l'istituto della remissione delle censure inteso come atto amministrativo, concesso da parte della competente autorità, in grado di fare cessare in capo al reo gli effetti della pena canonica ${ }^{81}$. A differenza di quanto avveniva nel CIC17 l'utilizzo del termine remissio adottato dal legislatore del 1983 vuole rimarcare la differenza esistente tra l'atto di natura giuridica e quello di natura sacramentale definito invece absolutio ${ }^{82}$.

Le nuove disposizioni si concentrano sui soggetti competenti a rimettere le pene, distinguendo tra le pene ordinarie e quelle riservate alla Sede Apostolica la cui eventuale riserva deve essere interpretata in senso stretto (can. 1054, § 3). Nel caso di pena inflitta o dichiarata stabilita dalla legge questa potrà essere rimessa, in via alternativa: a) dall'ordinario che ha promosso il giudizio penale o che ha, personalmente o tramite altri, inflitto la pena (can. 1355, $\S 1,1^{\circ}$ ); b) dall'ordinario del luogo in cui si trova il delinquente consultato $1^{\prime}$ ordinario che ha promosso il giudizio o inflitto la pena (can. 1355, $\S 1,2^{\circ}$ ), fatte salve, ovviamente, circostanze straordinarie che rendano impossibile tale consultazione. Nel caso di pene latae sententiae non ancora dichiarate e stabilite per legge esse potranno esser rimesse dall'ordinario ai propri sudditi, a coloro che si trovino nel territorio di sua competenza o che in esso abbiano commesso il delitto. In questo caso una competenza residuale è concessa anche a qualunque vescovo (anche titolare) ma nel solo atto della confessione sacramentale.

${ }^{80}$ In questo senso si vedano: A. BORRAS, Les sanctions dans l'Église, cit., pp. 125-128; V. DE PAOLIS, La cessazione delle pene (cann. 1354-1363), cit., p. 496.

81 Cfr. D. CITO, La remissione della pena canonica, cit., p. 117, e B.F. PIGHIN, Diritto penale canonico, cit., p. 275.

82 Cfr. G. DI MATTIA, Sostanza e forma nel nuovo diritto penale canonico, in Il nuovo Codice di Diritto Canonico novità, motivazione e significato, Libreria Editrice Pontificia Università Lateranense, Roma, 1983, pp. 409-437, in particolare p. 428. Si vedano in proposito i cann. 508, § 1, 566, § 2,976 . 
Gli stessi soggetti già individuati dal can. 1355 potranno rimettere una pena latae o ferendae sententiae stabilita da un precetto che non sia stato dato dalla Sede Apostolica, ma in questi casi dovrà essere previamente consultato l'autore del precetto, salvo che ciò non sia reso impossibile da circostanze straordinarie (can. 1356).

Oltre ai soggetti indicati nei cann. 1355-1356 la pena potrà essere rimessa da tutti i soggetti che possiedano la potestà per dispensare da una legge munita di pena o per liberare da un precetto che commina una pena. Tale specifica potestà potrà essere attribuita anche ad altri soggetti per legge o precetto (can. 1354). Come si vede il CIC83 ha notevolmente ampliato il novero dei soggetti competenti a rimettere la censura e ciò nell'ottica di consentire al reo di ottenere la liberazione dagli effetti previsti dalla sanzione penale con la maggiore facilità possibile ${ }^{83}$.

La Sede Apostolica potrà sempre riservare a sé o ad altri soggetti la remissione della pena (can. 1354, § 3) ma tale riserva potrà essere stabilita solamente prima dell'inflizione o dichiarazione della pena dal momento che il CIC83 non ha ripreso la distinzione tra riserva a iure e $a b$ homine contenuta nel can. 2242, § 2, CIC1784.

Il CIC83 ha ribadito la disposizione tradizionale riguardante i requisiti soggettivi richiesti al reo per ottenere la remissione della censura, confermando che è necessario il recesso dalla contumacia da parte di quest'ultimo, da provarsi mediante un vero pentimento per il delitto compiuto e la congrua riparazione - o almeno il serio proposito di riparazione - dei danni o dello scandalo provocati con la propria condotta (can. 1347, § 2). In presenza del recesso dalla contumacia sarà anzi obbligatorio concedere la remissione della pena a norma di quanto stabilito dal can. 1358, § 1 , sebbene chi rimetta la censura possa provvedere ad ammonire il reo o comunque a imporgli un rimedio penale (can. 1348) o una penitenza (can. 1358, § 2). Tali requisiti si pongono in linea di continuità con quanto stabilito in linea generale per l'applicazione delle pene. Il can. 1341 prescrive, infatti, che l'ordinario provveda ad

${ }^{83}$ Norme tutte sostanzialmente confermate anche nelle disposizioni contenute nella costituzione Pascite gregem Dei del 23 maggio 2021. Cfr. V. DE PAOLIS, D. CITO, Le sanzioni nella Chiesa, cit., pp. 264-266 e R. MAZZOLA, La pena latae sententiae nel diritto canonico. Profili comparati di teoria generale, cit., pp. 325-328.

${ }^{84}$ Molto opportunamente Cito fa inoltre notare che la riserva circa la remissione di una pena è soggetta a interpretazione stretta e dunque: "non solo occorre che la fattispecie delittuosa sia esattamente quella prevista, ma anche che sarà riservata soltanto la pena esplicitamente stabilita come riservata e non altre eventualmente applicate in suo luogo", D. CITO, La remissione della pena canonica, cit., p. 127. 
avviare un procedimento penale (giudiziale o amministrativo) solo se con altre modalità non si sia ottenuta una sufficiente riparazione dello scandalo, il ristabilimento della giustizia e l'emenda del reo, circostanze che rappresentano il vero obiettivo del diritto penale canonico e che, all'atto della remissione, dovranno essere opportunamente valutati. Accanto al recesso dalla contumacia il codice prevede che non si possa concedere la remissione qualora questa sia stata estorta per timore grave (can. 1360) $)^{85}$.

Il provvedimento assolutorio dovrà in ogni caso specificare le pene alle quali fa riferimento e avrà efficacia solamente con riferimento a tali pene, a meno che non intervenga una remissione generale in grado di rimettere al delinquente tutte le pene a eccezione di quelle dolosamente non dichiarate (can. 1359).

Dal momento che la remissione non è sovrapponibile alla assoluzione dai peccati, potrà essere concessa anche a persona assente $\mathrm{o}$, comunque, sotto condizione (can. 1361, § 1) sia risolutiva che sospensiva ${ }^{86}$. Il codice prevede che il provvedimento di remissione in foro esterno sia dato per iscritto "a meno che una grave causa suggerisca altrimenti" (can. $1361, \S 2$ ); in questo caso la grave causa andrà valutata con particolare attenzione dal momento che il reato è comunque noto, essendo avvenuto nel foro esterno, e perciò anche la remissione dovrà godere della medesima pubblicità. Una particolare circostanza da valutare sarà certamente quella del tempo intercorso tra $\mathrm{i}$ fatti delittuosi e il provvedimento di remissione, la cui pubblicità potrebbe rievocare nella comunità ecclesiale vicende oramai sopite.

In ogni caso il can. 1361, § 3, prevede che non si dia particolare pubblicità alla domanda di remissione né allo stesso provvedimento a meno che ciò non sia utile a tutelare la buona fama del reo o si renda necessario per la riparazione dello scandalo. Tale disposizione assumerà particolare rilevanza nei casi di delitti occulti o di delitti noti a una cerchia ristretta di fedeli per i quali la eccessiva pubblicità potrebbe creare nocumento al reo o alla stessa comunità.

\section{8 - (segue) la remissione delle censure in foro interno}

\footnotetext{
85 La nuova formulazione del can. 1360 prevede la invalidità della remissione estorta non soltanto mediante timore grave, ma anche per mezzo della forza o per dolo.

86 Cfr. V. DE PAOLIS, La cessazione delle pene (cann. 1354-1363), cit., p. 504.
} 
Al fine di ovviare al problema della eccessiva commistione tra i due fori, la nuova codificazione ha previsto che, in via ordinaria, la remissione delle censure debba avvenire in foro esterno, anche se determinate circostanze possono suggerire che la pena venga rimessa in foro interno. Si è già detto della competenza di qualunque vescovo di rimettere le pene latae sententiae, non ancora dichiarate e non riservate, nel sacramento della confessione e, quindi, nel solo foro interno sacramentale (can. 1355, § 2). Tale facoltà riguarda anche il canonico penitenziere (can. 508) e il cappellano negli ospedali, nelle carceri e nei viaggi in mare; quest'ultimo però, stante il dettato del can. $566, \S 2$, non sembra avere tale facoltà limitatamente al solo foro interno sacramentale, potendo evidentemente rimettere le censura anche nel foro interno extrasacramentale ${ }^{87}$. Nel corso del Giubileo straordinario della Misericordia del 2016 è stata concessa la speciale facoltà di assolvere i peccati riservati alla Sede Apostolica anche ai Missionari della misericordia, sacerdoti specificatamente incaricati ad amministrare il sacramento della riconciliazione nel corso dell'anno giubilare $^{88}$. A fare data dal 2016 è stata estesa a tutti i sacerdoti la facoltà "absolvendi peccatum eorum qui abortum procuraverunt"89; facoltà che può essere esercitata nel solo foro interno sacramentale ${ }^{90}$.

87 La maggiore ampiezza della facoltà concessa ai cappellani è giustificata dalle particolari circostanze e luoghi in cui essi si trovano a svolgere il loro ministero. De Paolis ritiene che tale facoltà sia esercitabile dai cappellani anche in foro esterno cfr.: V. DE PAOLIS, Quaestiones miscellaneae, in Periodica, 74 (1985), pp. 473-474, in senso contrario D. CITO, La remissione della pena canonica, cit., pp. 132-133.

88 FRANCESCO, Bolla d'indizione del Giubileo straordinario della Misericordia Misericordiae vultus, 11 aprile 2015, n. 18, in AAS 107 (2015), pp. 399-420, ivi p. 413. Tale facoltà è stata concessa per il medesimo periodo anche ai Penitenzieri delle Basiliche papali e ai sacerdoti componenti della Penitenzieria Apostolica, cfr. J.I. ARRIETA, Censure, irregolarità e impedimenti all'attenzione del Confessore e del Penitente, in XXVII Corso sul foro interno, Palazzo della Cancelleria 29 febbraio-4 marzo 2016, in http://www.penitenzieria.va/content/penitenzieriaapostolica/it/profilo/eventi.html [accesso: 31 luglio 2021] p. 11.

${ }^{89}$ FRANCESCO Lettera apostolica Misericordia et misera, 20 novembre 2016, n. 12, in AAS 108 (2016), pp. 1311-1327, ivi pp. 1319-1320.

${ }_{90}$ PONTIFICIO CONSIGLIO PER I TESTI LEGISLATIVI, Lettera del 29 novembre 2016 (Prot. n. 15675/2016 rinvenibile in http://www.delegumtextibus.va/ content/testilegislativi/it/risposte-particolari/codex-iuris-canonici.html [accesso: 31 luglio 2021]). Cfr. sul punto: J.I. ARRIETA, Le censure canoniche: identità e prospettive per una corretta pastorale. Irregolarità e impedimenti all'attenzione del confessore e del penitente, in XXX Corso sul foro interno, 25-29 marzo 2019 Roma-Palazzo della Cancelleria, in http://www.penitenzieria.va/content/penitenzieriaapostolica/it/profilo/eventi.html [accesso: 31 luglio 2021]. 
Il can. 1357 stabilisce invece la facoltà, in capo al confessore, di concedere la remissione in foro interno sacramentale limitatamente alle censure latae sententiae di scomunica o interdetto non dichiarate. Tale facoltà non riguarda i casi di pericolo di morte, già disciplinati ex can 976 né va a intaccare la facoltà ordinariamente concessa al canonico penitenziere ai sensi del can. 508, ma riguarda i casi in cui al penitente "durum sit in statu gravis peccati permanere" per il tempo necessario a che il superiore competente provveda alla remissione della censura. In questi specifici casi il confessore dovrà imporre al penitente l'onere di ricorrere, entro un mese, al soggetto ordinariamente competente a rimettere la censura e di attenersi poi alle decisioni di quest'ultimo, pena la reincidenza nella censura. Contemporaneamente il confessore dovrà imporre una congrua penitenza e la riparazione dello scandalo e del danno qualora ve ne sia urgenza (can. 1357, § 2). Tali ultime prescrizioni andranno applicate anche a coloro che avevano ricevuto la remissione in pericolo di morte ai sensi del can. 976, una volta che si siano rimessi in salute (can. 1357, § 3) ${ }^{91}$.

Il can. 1357 è stato inserito nella vigente codificazione per consentire, a quanti fossero incorsi nella scomunica o nell'interdetto, di potere comunque accedere, in determinati casi, ai sacramenti dell'assoluzione sacramentale e dell'unzione degli infermi anche prima della remissione della relativa censura, riprendendo di fatto il dettato del can. 2254 CIC1792.

Secondo quanto sostenuto da Cito, in base alla vigente codificazione, sembrerebbe non più possibile ottenere la remissione in foro interno sacramentale di pene ferendae sententiae o latae sententiae dichiarate, a meno che tale remissione non avvenga in caso di pericolo di morte ai sensi del can. 97693. La contestuale mancata riproposizione della

91 Si vedano sul punto le precisazioni contenute nella risposta data dal Segretario del Pontificio Consiglio per i Testi Legislativi con Lettera dell'8 aprile 2014 (Prot. n. 14452/2014) rinvenibile in http://www.delegumtextibus.va/content/testilegislativi/it/risposteparticolari/codex-iuris-canonici.html [accesso: 31 luglio 2021]) nella quale sostanzialmente si riprende il contenuto del can. 1357.

92 F.E. ADAMI, Continuità e variazioni di tematiche penalistiche nel nuovo Codex iuris canonici, cit., pp. 106-113; P. CIPROTTI, Qualche punto caratteristico della riforma del diritto penale canonico, cit., p. 141; M. VENTURA, Pena e penitenza nel diritto canonico postconciliare, cit., pp. 101-105; D. CITO, La remissione della pena canonica, cit., pp. 133-134; V. DE PAOLIS, D. CITO, Le sanzioni nella Chiesa, cit., pp. 270-272; B.F. PIGHIN, Diritto penale canonico, cit., p. 277.

93 D. CITO, La remissione della pena canonica, cit., pp. 136. 
disposizione contenuta nel can. 2251 del CIC17 fa sì che oggi nessun superiore possa esigere in foro esterno l'esecuzione della censura già rimessa in foro interno. Tuttavia la questione sembra sia stata risolta solo apparentemente, dal momento che rimarrà comunque aperto il problema di dare dimostrazione, in foro esterno, della remissione ottenuta nel foro interno sacramentale. Arias, a commento del can. 1355, § 2, sulla scorta di quanto già fatto dalla dottrina precedente (Cappello e Roberti), propone di risolvere la questione mediante "un certificato del vescovo che ha rimesso la pena [nel foro interno sacramentale], perché produca effetti in questo foro [esterno]", ritenendo che ciò non violi "l'obbligo del segreto sacramentale"; e concludendo che "la remissio di cui parliamo è un atto giuridico pubblico che si realizza nell'ambito sacramentale, ma nel foro esterno - proprio del diritto - con carattere occulto". Pur apprezzando lo sforzo prodotto per armonizzare i due fori nei casi di remissione, non sembra che ciò risolva del tutto i problemi di confusione tra di essi ${ }^{94}$.

Ammesso e non concesso, infatti, che non violi il segreto confessionale, (eliminare la precedente virgola) un documento rilasciato dal vescovo (o da un confessore il quale dia conto della remissione di una censura ottenuta in foro interno sacramentale), rimane comunque aperto il problema delle conseguenze nel foro esterno di una remissione ottenuta in ambito sacramentale, specie nel caso in cui il penitente non dia corso alle disposizioni di cui al can. 1357, §\$ 2 e 3, che prevedono specifici termini e condizioni per rivolgersi al superiore competente al fine di ottenere la definitiva remissione della censura. La dottrina si è interrogata sulla natura giuridica di tali condizioni, ovvero se esse siano di natura risolutiva o sospensiva: in che modo, il mancato rispetto delle condizioni imposte dal confessore che ha rimesso la censura in foro interno sacramentale, andrebbe a incidere sulla pena rimessa? Il dubbio non riguarda il carattere dell'assoluzione sacramentale, che sarebbe in ogni caso validamente conferita, quanto piuttosto il valore della remissione ${ }^{95}$. Per quanti hanno sostenuto il carattere risolutivo della remissione, in caso di mancato ricorso al superiore competente interverrebbe una nuova pena, per chi ne

${ }_{94}$ J. ARIAS, Commento al can. 1355, in Codice di diritto canonico e leggi complementari commentato, a cura di J.I. ARRIETA, Coletti a San Pietro, Roma, 2010, 3a ed., p. 905. Critico nei confronti della posizione assunta da Arias è A. BORRAS, Titulus VI De poenarum cessatione. c. 1355 comentario, in Comentario Exegético al Código de derecho canónico, cit., vol. IV/1, p. 439.

95 Già S. Tommaso distingueva tra la pena eterna, che viene rimessa mediante l'assoluzione dei peccati e la pena temporale che può permanere anche dopo la confessione: S. TOMMASO D'AQUINO, Summa theologiae III, q. 86, a. 4-5. 
ha invece sostenuto il carattere sospensivo, il mancato ricorso implicherebbe una reviviscenza della pena già rimessa ${ }^{96}$. Ritengo che il problema debba essere inquadrato nell'ottica dell'assoluzione, piuttosto che in quello della remissione, motivo per cui il mancato rispetto degli oneri imposti dal confessore ex can. 981 comporterà la perdita degli effetti normalmente conseguenti all'assoluzione sacramentale ${ }^{97}$. Alla luce di tale inquadramento appare più corretta la posizione di quanti hanno sostenuto il carattere sospensivo della remissione concessa in questi casi, per cui il mancato rispetto dell'obbligo di rivolgersi al competente superiore comporterà un'automatica riacquisizione di efficacia della censura precedentemente inflitta, al netto di eventuali giuste cause che abbiano impedito il rispetto dell'obbligo per motivi indipendenti dalla volontà del reo.

Rimane infine aperto il problema dei casi di censure latae sententiae non dichiarate ma originate da fatti non occulti. In questi casi il reo potrà, infatti, ottenere la remissione in foro interno sacramentale pur in presenza di un delitto potenzialmente noto a terzi, creando così una possibile commistione tra fori. Una soluzione si sarebbe potuta ottenere imponendo non tanto il ricorso al superiore competente, quanto piuttosto in foro interno extrasacramentale, così da potere provare l'avvenuta remissione una volta che la censura divenisse conoscibile in foro esterno; aggiustamento peraltro suggerito in molti casi dallo stesso confessore ${ }^{98}$.

\section{9 - Le modifiche introdotte dalla costituzione Pascite gregem Dei}

96 Riferisce del dibattito M. VENTURA, Pena e penitenza nel diritto canonico postconciliare, cit., pp. 103-104. Tra i primi vi sarebbero Wernz, Cappello, Bride e Arias, tra i secondi Michiels, Jombart, Borras e Adami.

97 Cfr. E. FRANK, I sacramenti dell'iniziazione, della penitenza e dell'unzione degli infermi. Commento ai canoni 834-1007 del Codice di Diritto Canonico, Urbaniana University Press, Città del Vaticano, 2018, 2a ed., p. 184.

98 Già prima dell'avvio dei lavori di riforma del CIC17 si era proposto di risolvere la questione scindendo il momento dell'assoluzione dal peccato da quello della remissione dalla censura, per la cessazione dei cui effetti si sarebbe dovuto comunque ricorrere al foro esterno: R. CASTILLO LARA, Algunas reflexiones sobre la futura reforma del libro $V$ C.I.C., cit., p. 338, nota 97. 
La recente costituzione apostolica Pascite gregem Dei99, con la quale è stato integralmente riformato il libro VI del codice di diritto canonico, ha introdotto alcune modifiche anche in materia di remissione delle censure.

Rispetto ad altre parti del libro VI, le modifiche in tema di remissione sono poche e di natura piuttosto puntuale, se si eccettuano le disposizioni introdotte in tema di recesso dalla contumacia al fine dell'ottenimento della remissione. Il legislatore, infatti, si è limitato per la più parte a riformulare i canoni esistenti al fine di rendere più chiaro il contenuto di alcune disposizioni già presenti nella codificazione del 1983.

Il can. 1355, nell'individuare i soggetti competenti a rimettere la pena stabilita per legge, specifica ora che si tratta di pena "ferendae sententiae irrogata vel latae sententiae declarata" laddove il canone previgente non specificava la tipologia di pena. Il can. 1356, con riferimento ai soggetti competenti a rimettere la pena stabilita con precetto indica ora, al primo posto tra tali soggetti, l'autore del precetto.

Il nuovo libro VI lascia sostanzialmente invariate le disposizioni relative alla remissione delle censure in foro interno, non risolvendo alcuno dei problemi sui quali la dottrina si è interrogata sin dalla codificazione del 1917. Il can. 1357, infatti, viene riproposto senza apprezzabili variazioni se si eccettua la correzione introdotta al $\S 3$; nel caso di penitente assolto da censura in periculo mortis da un qualunque sacerdote ex can. 976, l'onere per il penitente di ricorrere entro un mese all'autorità competente a rimettere la censura scatta "cessante periculo" e non più a seguito del generico ristabilimento in salute di cui alla precedente formulazione.

Le modifiche più consistenti riguardano, come detto, il recesso dalla contumacia necessario per l'ottenimento della remissione della pena. Il can. 1358, § 1, infatti, ribadisce che il recesso si possa provare solamente con il vero pentimento e con la contestuale congrua riparazione allo scandalo o al danno o con, almeno, il serio proposito di realizzare tale riparazione (can. 1347, § 2) ${ }^{100}$. La novità è rappresentata dal richiamo al nuovo $\S 4$ del can. 1361, nel quale si prevede che l'ordinario, secondo il

99 Per volontà dello stesso pontefice la costituzione è stata promulgata mediante pubblicazione su L'Osservatore Romano, 161-122 (1 giugno 2021), pp. 2-3, ed entrerà in vigore il giorno 8 dicembre 2021.

$100 \mathrm{Il}$ nuovo can. 1347, § 2, peraltro, significativamente antepone la congrua reparatio scandali alla reparatio damni per significare l'importanza della evitatio scandali per l'ordinamento canonico, certamente prevalente rispetto alla pur importante necessità di riparazione del danno causato. 
suo prudente giudizio, "remissio dari non debet" finché il reo non abbia riparato il danno eventualmente conseguente al suo atto criminoso.

Il legislatore ha inoltre previsto, nel menzionato can. 1361, § 4, che, al fine di sollecitare la riparazione del danno causato, il reo possa essere ulteriormente sanzionato con le pene espiatorie previste dal can. 1336, §§ 2-4, ovvero mediante specifiche ingiunzioni, proibizioni o privazioni. Le sanzioni appaiono di particolare gravità atteso che il riformato canone specifica che esse potranno essere inflitte anche a colui che abbia già receduto dalla contumacia senza tuttavia avere provveduto ad adeguato risarcimento.

Sebbene il tenore della norma appaia senza dubbio di estrema severità, non si può non accogliere favorevolmente la modifica volta a tutelare le parti lese, siano essi singoli o intere comunità di fedeli. La concessione della remissione in difetto di equa riparazione appariva senza dubbio lesiva dei diritti dei fedeli a ottenere giustizia rispetto a un torto subito e lasciava aperta la possibilità che il reo ricevesse la remissione senza avere provveduto all'effettiva riparazione dei danni causati. Il riferimento alla riparazione contenuto nel previgente can. 1357, § 2, era, infatti, riferito alle sole remissioni concesse in foro interno sacramentale e in casi di particolare urgenza, mentre oggi il dettato del $\S 4$ del can. $1361 \mathrm{fa}$ chiaramente intendere che la misura vada applicata sia alle censure rimesse in foro interno sacramentale che a quelle rimesse in foro esterno. In precedenza il mancato richiamo, nel can. 1358, § 1, alla necessità della riparazione rendeva tale imposizione certamente meno incisiva.

La misura è dunque maggiormente severa rispetto alle disposizioni precedentemente in vigore le quali non prevedevano il divieto di concedere la remissione sino alla avvenuta riparazione del danno né stabilivano pene atte a sanzionare la mancata riparazione da parte del reo.

\section{0 - Brevi considerazioni conclusive}

Come si è visto il vigente libro VI del codice di diritto canonico, pur avendo notevolmente semplificato le norme relative alla remissione, si pone in linea di continuità con la tradizione canonistica in materia di assoluzione dalle censure. Se da un lato, infatti, il legislatore ha inteso limitare al minimo le riserve di competenza in materia di concessione della remissione, ampliando di conseguenza il novero dei soggetti abilitati a rimettere le pene, dall'altro ha comunque mantenuto alcuni punti fermi fatti propri sin dalle prime elaborazioni sistematiche dell'istituto. Permane sino a oggi la difficoltà di scindere completamente quanto avviene nel foro 
esterno e quanto invece avviene nel foro interno sacramentale dal momento che, in sede di lavori di riforma, si sono volute mantenere ferme alcune tipicità dell'ordinamento: l'impossibilità per il soggetto colpito da censura o interdetto di ottenere la remissione dei peccati e la conseguente possibilità di ottenere la remissione della censura anche nell'ambito del sacramento della confessione per non compromettere il fine salvifico dell'ordinamento canonico. Sin dalle sue prime formulazioni l'istituto dell'assoluzione dalle censure si è posto al confine tra l'ambito dell'esercizio della potestas iurisdictionis in foro esterno e in foro interno, motivo per cui permane sino a oggi una difficoltà legata al fatto di scindere completamente quanto avviene nell'uno piuttosto che nell'altro foro. Le recentissime riforme operate da papa Francesco non hanno risolto tali problematiche, sebbene abbiano ulteriormente chiarito il senso di alcune norme. L'attenzione posta al tema della riparazione del danno va senza dubbio accolta positivamente e costituisce un equo contemperamento tra il diritto del reo a ottenere la remissione della pena in caso di recesso dalla contumacia e quello dei soggetti colpiti dal comportamento criminoso a essere in qualche modo reintegrati nei loro diritti. Con ciò si offre maggiore tutela ai soggetti deboli e alle loro comunità di appartenenza, ingiustamente colpiti dal comportamento criminale, pur senza pregiudicare il fine medicinale della pena, tipico dell'ordinamento penale canonico, volto a consentire un pieno recupero del delinquente. 